\title{
Cancellation of fluctuation in stochastic ranking process with space-time dependent intensities,
}

\author{
Tetsuya Hattori \\ Laboratory of Mathematics, Faculty of Economics, Keio University, \\ Hiyoshi Campus, 4-1-1 Hiyoshi, Yokohama 223-8521, Japan \\ URL: http://web.econ.keio.ac.jp/staff/hattori/research.htm \\ email: hattori@econ.keio.ac.jp
}

January 10, 2018

\begin{abstract}
We consider the stochastic ranking process with space-time dependent unbounded jump rates for the particles. We prove that the joint empirical distribution of jump rate and scaled position converges almost surely to a deterministic distribution in the infinite particle limit. We assume topology of weak convergence for the space of distributions, which implies that the fluctuations among particles with different jump rates cancel in the limit. The results are proved by first finding an auxiliary stochastic ranking process, for which a strong law of large numbers is applied, and then applying a multi time recursive Gronwall's inequality. The limit has a representation in terms of non-Markovian processes which we call point processes with last-arrival-time dependent intensities.
\end{abstract}

2000 Mathematics Subject Classification. Primary 60K35; Secondary 82C22.

Key words. Stochastic ranking process, hydrodynamic limit, law of large numbers, complete convergence, last-arrival-time dependent intensity, Gronwall inequality. 


\section{Introduction.}

Let $N$ be a positive integer and $T>0 . T$ is an arbitrary constant fixed throughout the paper, and we are interested in the limit $N \rightarrow \infty$. A stochastic ranking process is a stochastic system of $N$ particles on a line segment $[0,1]$ for a time interval $[0, T]$, defined as follows. Let $W \subset C^{1}([0,1] \times[0, T] ;[0, \infty))$ be a set of non-negative valued $C^{1}$ functions in two variables such that the partial derivative with respect to the first variable is bounded on $[0,1] \times[0, T]$. We write

$$
\|f\|_{\mathrm{T}}=\sup _{(z, s) \in[0,1] \times[0, T]}|f(z, s)|
$$

for a function $f:[0,1] \times[0, T] \rightarrow \mathbb{R}$, and put

$$
C_{W}=\sup _{w \in W}\left\|\frac{\partial w}{\partial y}\right\|_{\mathrm{T}}<\infty .
$$

Let $w_{1}, w_{2}, \ldots$ be an infinite sequence in $W$.

Let $y_{1}^{(N)}, y_{2}^{(N)}, \ldots, y_{N}^{(N)}$ be a permutation of $\left\{\frac{i}{N} \mid i=0,1, \ldots, N-1\right\}$. Then a stochastic ranking process is a system of stochastic processes $\left\{Y_{i}^{(N)} \mid i=1,2, \ldots, N\right\}$ defined on a probability space $(\Omega, \mathcal{F}, \mathrm{P})$ by

$$
\begin{aligned}
& Y_{i}^{(N)}(t)=y_{i}^{(N)} \\
& \quad+\frac{1}{N} \sum_{j=1}^{N} \int_{s \in(0, t]} \int_{\xi \in[0, \infty)} \mathbf{1}_{Y_{j}^{(N)}(s-)>Y_{i}^{(N)}(s-)} \mathbf{1}_{\xi \in\left[0, w_{j}\left(Y_{j}^{(N)}(s-), s\right)\right)} \nu_{j}^{(N)}(d \xi d s) \\
& \quad-\int_{s \in(0, t]} \int_{\xi \in[0, \infty)} Y_{i}^{(N)}(s-) \mathbf{1}_{\xi \in\left[0, w_{i}\left(Y_{i}^{(N)}(s-), s\right)\right)} \nu_{i}^{(N)}(d \xi d s), \\
& i=1,2, \ldots, N, t \geqq 0 .
\end{aligned}
$$

Here, $\mathbf{1}_{A}$ is the indicator function of an event $A$, and for each $N, \nu_{i}^{(N)}, i=1,2, \ldots, N$, are independent Poisson random measures on $[0, \infty) \times[0, \infty)$ with uniform unit intensity measures, i.e., $\mathrm{E}\left[\nu_{i}^{(N)}([a, b] \times[c, d])\right]=(b-a)(d-c)$ for $b>a \geqq 0$ and $d>c \geqq 0$, and $\nu_{i}^{(N)}(A)$ and $\nu_{i}^{(N)}(B)$ are independent Poisson variables if $A \cap B=\emptyset$.

If we put

$$
\tilde{\nu}_{i}^{(N)}(t)=\int_{s \in(0, t]} \int_{\xi \in[0, \infty)} \mathbf{1}_{\xi \in\left[0, w_{i}\left(Y_{i}^{(N)}(s-), s\right)\right)} \nu_{i}^{(N)}(d \xi d s),
$$

then (3), the position of the particle $i$ at time $t$, is expressed as

$$
Y_{i}^{(N)}(t)=y_{i}^{(N)}+\frac{1}{N} \sum_{j=1}^{N} \int_{0}^{t} \mathbf{1}_{Y_{j}^{(N)}(s-)>Y_{i}^{(N)}(s-)} \tilde{\nu}_{j}^{(N)}(d s)-\int_{0}^{t} Y_{i}^{(N)}(s-) \tilde{\nu}_{i}^{(N)}(d s) .
$$

We then see that the time development of $\left\{Y_{i}^{(N)}\right\}$ is determined by the move-to-front rules [17] driven by the point processes $\left\{\tilde{\nu}_{i}^{(N)}\right\}$ with space-time dependent intensities given by the 'density functions' $\left\{w_{i}\right\}$. If $w_{i}$ is constant then the process $\tilde{\nu}_{i}^{(N)}$ is the Poisson process, while in general, its increments depend on past and is dependent on other particles. The move-to-front rule in particular implies that the particle system $\left\{Y_{i}^{(N)}\right\}$ as a whole takes values in the rearrangement of $\frac{i}{N}, i=0,1, \ldots, N-1$. Each particle either increases its position by $\frac{1}{N}$, or else takes the value 0 , i.e. jumps to the top position, as $t$ increases. 
A starting point for our study is the joint empirical distribution of $w_{i}$ and the position, given by

$$
\mu_{t}^{(N)}=\frac{1}{N} \sum_{i=1}^{N} \delta_{\left(w_{i}, Y_{i}^{(N)}(t)\right)} .
$$

Here $\delta_{c}$ is a unit measure concentrated at $c$. (We will use this notation for a unit measure on any probability space.) $\mu^{(N)}$ is a stochastic process taking values in the set of Borel probability measures, with initial distribution being

$$
\mu_{0}^{(N)}=\frac{1}{N} \sum_{i=1}^{N} \delta_{\left(w_{i}, y_{i}^{(N)}\right)} .
$$

By considering a process $X_{i}^{(N)}(t)=N Y_{i}^{(N)}(t)+1$ taking values in positive integers, we see that a stochastic ranking process is a model of ranking system, such as the sales ranks found at online bookstores [5, 6, 4, 7, 9, 8, 15, 16, 13. As a model of popularity ranks of an online bookstore, the move-to-front rule defines the rank as a stochastic number with the 'latest purchased book as most popular' rule. That the intensity $w_{i}$ differs for different book $i$ represents that there are popular books and less popular ones. (Many books on mathematics perhaps provide examples of the latter.) See also [13] and references therein for more background.

We will prove existence of hydrodynamic limit, that assuming convergence of $\mu_{0}^{(N)}$ as $N \rightarrow \infty$ we have convergence of $\mu_{t}^{(N)}$ for all $t \in[0, T]$. The common standard quantities effective for the move-to-front rules are the characteristic curves $Y_{C}^{(N)}$ defined by

$$
\begin{aligned}
& Y_{C}^{(N)}(\gamma, t)=y_{0} \\
& +\frac{1}{N} \sum_{j=1}^{N} \int_{s \in\left(t_{0}, t\right]} \int_{\xi \in[0, \infty)} \mathbf{1}_{Y_{j}^{(N)}(s-) \geqq Y_{C}^{(N)}(\gamma, s-)} \\
& \times \mathbf{1}_{\xi \in\left[0, w_{j}\left(Y_{j}^{(N)}(s-), s\right)\right)} \nu_{j}^{(N)}(d \xi d s), \\
& \gamma=\left(y_{0}, t_{0}\right) \in[0,1] \times[0, T], t \geqq t_{0},
\end{aligned}
$$

and a set of spatial distribution functions $\varphi^{(N)}$ of $\mu_{t}^{(N)}$ defined by

$$
\begin{aligned}
& \varphi^{(N)}(d w, \gamma, t)=\mu_{t}^{(N)}\left(d w \times\left[Y_{C}^{(N)}(\gamma, t), 1\right]\right) \\
& \gamma=\left(y_{0}, t_{0}\right) \in[0,1] \times[0, T], t \geqq t_{0}, w \in W
\end{aligned}
$$

The latter is a refinement of the former in the sense that (5) and (8) imply

$$
\begin{aligned}
& Y_{C}^{(N)}(\gamma, t)=y_{0}+\frac{\left[N\left(1-y_{0}\right)\right]}{N}-\varphi^{(N)}(W, \gamma, t), \\
& \gamma=\left(y_{0}, t_{0}\right) \in[0,1] \times[0, T], t \geqq t_{0} .
\end{aligned}
$$

Since $\varphi^{(N)}$ determines $\mu_{t}^{(N)}$, the convergence problem of $\mu_{t}^{(N)}$ is reduced to that of $\varphi^{(N)}$.

If the intensity densities $w \in W$ are independent of $y$, then $\varphi^{(N)}$ is an arithmetic mean of independent stochastic processes, so that a strong law of large numbers for a sum of independent processes can be applied to prove existence of almost sure $N \rightarrow \infty$ limit under reasonable assumptions [4]. In contrast, when the intensity densities depend on $y$, the problem is a more involved one of law of large numbers for dependent processes. The case of spatially varying intensity densities was 
first studied in [13], where we found the existence of the limit under restricting assumptions. One assumption was boundedness of $W$, which excludes, for example, Pareto (power law) distributions which may be of interest in applying stochastic ranking processes to social studies [8, 9]. Another, a mathematically more essential assumption in [13] was that we adopted total variation norm for the topology of the space of Borel measures. This means that, among other points, the law of large numbers proved in [13] is a cancellation of fluctuations among processes $\left\{Y_{i}^{(N)}\right\}$ having the same associated intensity densities $w_{i}=w$. This assumption is to be compared with the results in [4], where we proved a corresponding convergence theorem (for the easier case of spatially constant intensity densities) with topology of the space of Borel measures induced by weak convergence, which, for example, allows all the $w_{i}$ 's to be different and the cancellation of fluctuation is still implied. It would be mathematically interesting to see how this law of large numbers (fluctuation cancellation) mechanism, which escaped from our hands in [13], would be stable against introduction of the spatial dependence of $w \in W$.

The essential ingredient of the proof of this paper may be summarized as follows:

- Discovery of the point process with last-arrival-time dependent intensity [10, 11]. The hydrodynamic limit turned out to have an expression in terms of probabilities of the process. This expression was absent in [13, which was a partial cause of a technical boundedness assumptions on $W$. The process lack independent increment properties, which is a remnant of stochastic dependence among the particles through the position dependence of $w \in W$. This is to be compared with the earlier studies for position independent $w$, where the corresponding quantities have explicit formulas using exponentials of integration of $w$, related to the probabilities of the Poisson process [5, 4].

- Discovery of an intermediate model, defined in $\S$ 3, which we call the flow driven stochastic ranking process. The 'intensity densities' for the flow driven stochastic ranking process are involved but without position dependence, hence the distribution function $\varphi$ for this model is a sum of independent processes, and a standard law of large numbers has a chance of explaining the fluctuation cancellation mechanism. (Introduction of an intermediate model resembles a notion of local equilibrium which appears in the hydrodynamic limit for diffusions. The limit of the stochastic ranking process is, in terms of fluid dynamics, a one-sided flow with evaporation from upper stream $y=0$ to the down stream $y=1$, and the correspondence to diffusion is only a kind of metaphor.)

Since the stochastic dependence among the particles induced by the move-to-front rule is handled by introduction of distribution functions $\varphi$, the real challenge from a viewpoint of mathematical analysis is the stochastic dependence through position dependence of $w \in$ $W$, which was first studied in [13]. In the reference we adopted a sophisticated method (than adopted in this paper) based on submartingale inequalities, which worked well with the restricting assumptions in the reference, and enabled a relatively quick proof without introducing intermediate models.

- Development and application of a uniform strong complete law of large numbers for independent monotone function valued random variables.

Since the flow driven stochastic ranking process is stochastically similar to the stochastic ranking process with position independent intensities, a strong law of large numbers for independent processes is applicable. Since, however, we later need to compare this intermediate model with the original model, we apply uniform convergence results stronger than in earlier works [4], where the law of large numbers for the independent processes was practically the final goal. 
- Application of a hierarchy of multi time Gronwall type inequalities. The last step is to prove that the original stochastic ranking process has a same limit with the (appropriately chosen) flow driven stochastic ranking process. To evaluate the difference, we couple the two models, and resort to a multi time variable recursive version of Gronwall type inequality which we develop in $\S 4$.

In [13], we also proved the occurrence of propagation of chaos. Namely, for each integer $L$, the tagged particle system

$$
\left(Y_{1}^{(N)}(t), Y_{2}^{(N)}(t), \ldots, Y_{L}^{(N)}(t)\right)
$$

converges to a limit process uniformly in $t \in[0, T]$ as $N \rightarrow \infty$, if the system of the initial positions $\left(y_{1}^{(N)}, y_{2}^{(N)}, \ldots, y_{L}^{(N)}\right)$ converges, and the components of the limit are independent of each other. A corresponding result is also proved in this paper.

The plan of the present paper is as follows. In $\S 2$ we state the main results, that the stochastic ranking process with space-time dependent intensities has a limit characterized by the point processes with last-arrival-time dependent intensities. For convenience, we will summarize the definition and relevant results of the point processes in $\S \mathrm{A}$. In $\S 3$ we formulate the flow driven stochastic ranking process and prove the existence of the strong uniform law of large numbers (the large particle number limit). In $\S$ we formulate and prove a hierarchy of a multi time version of the Gronwall's inequality, which we use in $\S 5$ to complete a proof of the main theorem stated in $\S 2$

\section{Formulation and the main results.}

To state the main results precisely, we first formulate the quantities which appear in the infinite particle limit of the stochastic ranking process. Denote the sets of initial $(t=0)$ points in the space-time $[0,1] \times[0, T]$, the set of upper stream boundary $(y=0)$ points, and their union, the set of initial/boundary points, respectively by

$$
\begin{aligned}
& \Gamma_{b}=\{(0, s) \mid 0 \leqq s \leqq T\} \\
& \Gamma_{i}=\{(z, 0) \mid 0 \leqq z \leqq 1\} \\
& \Gamma=\Gamma_{b} \cup \Gamma_{i}
\end{aligned}
$$

For $t \in[0, T]$, denote the set of initial/boundary points up to time $t$ by

$$
\Gamma_{t}=\left\{\left(z, t_{0}\right) \in \Gamma \mid t_{0} \leqq t\right\}=\Gamma_{i} \cup\left\{\left(0, t_{0}\right) \in \Gamma_{b} \mid 0 \leqq t_{0} \leqq t\right\},
$$

and the set of admissible pairs of the initial/boundary point $\gamma$ and time $t$ by

$$
\Delta_{T}=\left\{(\gamma, t) \in \Gamma_{T} \times[0, T] \mid \gamma \in \Gamma_{t}\right\} .
$$

Define the set of flows $\Theta_{T}$ on $[0,1] \times[0, T]$ by

$$
\begin{aligned}
\Theta_{T}=\{ & \theta: \Delta_{T} \rightarrow[0,1] \mid \text { continuous, } \theta\left(\left(y_{0}, t_{0}\right), t_{0}\right)=y_{0},\left(y_{0}, t_{0}\right) \in \Gamma_{T}, \\
& \text { surjective and non-increasing in } \gamma \text { for each } t, \\
& \text { non-decreasing in } t \text { for each } \gamma\},
\end{aligned}
$$

where, we define a total order $\succeq$ on the initial/boundary set $\Gamma_{T}$ by

$$
s \leqq t, z \leqq y \Leftrightarrow(0, T) \succeq(0, t) \succeq(0, s) \succeq(0,0) \succeq(z, 0) \succeq(y, 0) \succeq(1,0) .
$$


For example,

$$
\theta((1,0), t)=1, \quad t \in[0, T], \theta \in \Theta_{T} .
$$

For each $\theta \in \Theta_{T}, w \in W$, and $z \in[0,1]$, define $\tilde{w}_{\theta, w, z}$, a non-negative valued continuous function of $(s, t)$ satisfying $0 \leqq s \leqq t \leqq T$, by

$$
\tilde{w}_{\theta, w, z}(s, t)= \begin{cases}w(\theta((z, 0), t), t), & \text { if } s=0 \\ w(\theta((0, s), t), t), & \text { if } s>0 .\end{cases}
$$

Note that $\tilde{w}_{\theta, w, z}$ is independent of $z$ if $s>0$. Let $\theta \in \Theta_{T}$, and put

$$
\begin{aligned}
& \varphi_{\theta}(d w, \gamma, t)=\int_{z \in\left[y_{0}, 1\right]} \mathrm{P}\left[\tilde{\nu}_{\theta, w, z}(t)=\tilde{\nu}_{\theta, w, z}\left(t_{0}\right)\right] \mu_{0}(d w \times d z), \\
& \gamma=\left(y_{0}, t_{0}\right) \in \Gamma_{t}, \quad(\gamma, t) \in \Delta_{T}
\end{aligned}
$$

where $\tilde{\nu}_{\theta, w, z}$ is the point process with last-arrival-time dependent intensity, denoted by $N$ in $\S$ A with the 'intensity density' $\omega(s, t)$ in the definition (141) of the process given by $\tilde{w}_{\theta, w, z}(s, t)$ of (16), and $\mu_{0}$ is a Borel probability measure on the direct product space $W \times[0,1]$.

The following is proved in [10].

Theorem 1 ([10, Theorem 9, (89)]) There exists a unique flow $y_{C} \in \Theta_{T}$ such that

$$
\theta(\gamma, t)=1-\varphi_{\theta}(W, \gamma, t), \gamma=\left(y_{0}, t_{0}\right) \in \Gamma,(\gamma, t) \in \Delta_{T},
$$

holds for $\theta=y_{C}$.

In [10] below the Theorem it is remarked that there exists $\mu_{t}$, taking values in the space of Borel probability measures on $W \times[0,1]$, such that

$$
\begin{aligned}
& \varphi_{y_{C}}\left(d w,\left(y_{0}, t_{0}\right), t\right)=\mu_{t}\left(d w \times\left[y_{C}\left(\left(y_{0}, t_{0}\right), t\right), 1\right]\right), \\
& \left(\left(y_{0}, t_{0}\right), t\right) \in \Delta_{T},
\end{aligned}
$$

and below Theorem 1 in [10] it is also remarked that $\mu_{t}$ and $y_{C}$ satisfy

$$
y_{C}(\gamma, t)=y_{0}+\int_{t_{0}}^{t} \int_{W \times\left[y_{C}(\gamma, s), 1\right)} w(z, s) \mu_{s}(d w \times d z) d s .
$$

Next we state our assumptions on the infinite particle limit $N \rightarrow \infty$ of initial $(t=0)$ conditions. We will assume a standard supremum norm on the space of continuous functions on the closed interval $[0,1] \times[0, T]$, with which we define the weak convergence of Borel probability measures on $W$ in the standard way [1]. For the initial distribution $\mu_{0}^{(N)}$ in (6), we assume the following weak convergence with additional uniform bounds on the order of convergence to $\mu_{0}$ in (17):

For any set $H \subset C^{0}(W \times[0,1] ; \mathbb{R})$ of uniformly bounded, equicontinuous functions, $\exists \delta \in\left(0, \frac{1}{2}\right), \exists C>0$;

$$
\begin{aligned}
& (\forall N \in \mathbb{N})(\forall \tilde{h} \in H)(\forall y \in[0,1]) \\
& \left|\int_{W \times[y, 1]} \tilde{h}(w, z) \mu_{0}^{(N)}(d w \times d y)-\int_{W \times[y, 1]} \tilde{h}(w, z) \mu_{0}(d w \times d y)\right| \leqq \frac{C}{N^{\delta}} .
\end{aligned}
$$

Denote the marginal distribution of $\mu_{0}$ on $W$ by $\lambda$;

$$
\lambda(d w)=\mu_{0}(d w \times[0,1]),
$$


and put

$$
\lambda^{(N)}=\frac{1}{N} \sum_{i=1}^{N} \delta_{w_{i}} .
$$

Comparing with (5), we see that $\lambda^{(N)}$ is the marginal distribution of $\mu_{t}^{(N)}$ on $W$ for all $t$;

$$
\lambda^{(N)}(d w)=\mu_{t}^{(N)}(d w \times[0,1]), t \in[0, T] .
$$

For $\lambda$ we assume

$$
M_{W}:=\int_{W}\|w\|_{\mathrm{T}} \lambda(d w)<\infty .
$$

The assumption (21) implies, with (24) and (22),

$$
\lambda^{(N)} \rightarrow \lambda \text {, weakly as. } N \rightarrow \infty .
$$

In addition we assume convergence of the average of $\|w\|_{\mathrm{T}}$ :

$$
\lim _{N \rightarrow \infty} \int_{W}\|w\|_{\mathrm{T}} \lambda^{(N)}(d w)=M_{W}
$$

Remark. In (21) we assume uniform order of convergence $O\left(N^{-\delta}\right)$, while Ascoli-Arzelà type theorem implies uniform convergence but has no control in general on the order of convergence.

We are ready to state the main results of this paper.

Theorem 2 Under the assumptions (2), (21), (25), and (27), with probability 1, $\mu_{t}^{(N)} \rightarrow \mu_{t}$, weakly as $N \rightarrow \infty$, uniformly in $t$, where $\mu_{t}$ is as in (19). Explicitly, we prove

$$
\lim _{N \rightarrow \infty} \sup _{t \in[0, T]}\left|\int_{W} h(w) \mu_{t}^{(N)}(d w \times[y, 1])-\int_{W} h(w) \mu_{t}(d w \times[y, 1])\right|=0, \text { a.s. },
$$

for all $y \in[0,1]$ and bounded continuous function $h: W \rightarrow \mathbb{R}$.

Assume in addition that for a positive integer $L$ and $y_{i} \in[0,1), i=1,2, \ldots, L$,

$$
\nu_{i}^{(N)}=\nu_{i}, N \in \mathbb{N}, \text { and } \lim _{N \rightarrow \infty} y_{i}^{(N)}=y_{i}, \text { for } i=1,2, \ldots, L .
$$

Then, with probability 1 , the tagged particle system

$$
\left(Y_{1}^{(N)}(t), Y_{2}^{(N)}(t), \ldots, Y_{L}^{(N)}(t)\right)
$$

converges as $N \rightarrow \infty$, uniformly in $t \in[0, T]$ to a limit $\left(Y_{1}(t), Y_{2}(t), \ldots, Y_{L}(t)\right)$. Here, for each $i=1,2, \ldots, L, Y_{i}$ is the unique solution to

$$
\begin{aligned}
& Y_{i}(t)=y_{i}+\int_{s \in(0, t]} \int_{(w, z) \in W \times\left[Y_{i}(s-) .1\right]} w(z, s) \mu_{s}(d w \times d z) d s \\
& \quad-\int_{s \in(0, t]} \int_{\xi \in[0, \infty)} Y_{i}(s-) \mathbf{1}_{\xi \in\left[0, w_{i}\left(Y_{i}(s-), s\right)\right)} \nu_{i}(d \xi d s), \\
& i=1,2, \ldots, L, t \in[0, T] .
\end{aligned}
$$


Theorem 2 implies propagation of chaos for the stochastic ranking processes. For each $N$ all of $\left\{Y_{i}^{(N)}\right\}$ are random and interact with each other and $\mu_{t}^{(N)}$ is also random. However, the limit $\mu_{t}$ is deterministic. Furthermore, the randomness of the limit process $Y_{i}$ of a tagged particle depends only on its associated Poisson random measure $\nu_{i}$, and is independent of $Y_{j}$ or $\nu_{j}$ with $j \neq i$.

Incidentally, we proved almost sure convergence for $\mu_{t}^{(N)}$ in (28), while we made no assumptions on the relation between the set of measures $\left\{\nu_{i}^{(N)} \mid i=1,2, \ldots, N\right\}$ for different $N$. This is stronger than the law of large numbers appearing in the context of random walks $\frac{1}{N} \sum_{i=1}^{N} X_{i}$, where each $X_{i}$ is fixed for all $N$, while (28) corresponds to considering $\frac{1}{N} \sum_{i=1}^{N} X_{i}^{(N)}$, where we assume no relation among $X_{i}^{(N)}$ for different $N$. Such a type of convergence is known for sums of real valued random variables as complete convergence [14, 2, 3]. In this context, (19) is an example of complete convergence for a sequence of measure valued random variables.

\section{Infinite particle limit of flow driven stochastic ranking process.}

\subsection{Flow driven stochastic ranking process.}

In this section, we introduce an intermediate model which we use to prove convergence of $\mu_{t}^{(N)}$ in Theorem 2,

Let $\left\{w_{i}\right\},\left\{y_{i}^{(N)}\right\}$, and $\left\{\nu_{i}^{(N)}\right\}$ be as in the stochastic ranking process (77). Let $\theta \in \Theta_{T}$ be a flow, and for each $i=1,2, \ldots, N$, let $\tilde{\nu}_{i}^{(N, \theta)}$ be a point process of $N$ in (147), with $\nu=\nu_{i}^{(N)}$ and $\omega=\tilde{w}_{\theta, w_{i}, y_{i}^{(N)}}$, where the last notation is as in (16) with $w=w_{i}$ and $z=y_{i}^{(N)}$. Define a system of stochastic processes $Y_{i}^{(N, \theta)}, i=1,2, \ldots, N$, by

$$
\begin{aligned}
& Y_{i}^{(N, \theta)}(t) \\
& =y_{i}^{(N)}+\frac{1}{N} \sum_{j=1}^{N} \int_{s \in(0, t]} \mathbf{1}_{Y_{j}^{(N, \theta)}(s-)>Y_{i}^{(N, \theta)}(s-)} \tilde{\nu}_{j}^{(N, \theta)}(d s) \\
& \quad-\int_{s \in(0, t]} Y_{i}^{(N, \theta)}(s-) \tilde{\nu}_{i}^{(N, \theta)}(d s), \quad i=1,2, \ldots, N, t \geqq 0 .
\end{aligned}
$$

We will call this system, the stochastic ranking process driven by the flow $\theta$.

The fluctuation of (31) is coupled to those of the stochastic ranking process of (3) via the set of Poisson random measures $\left\{\nu_{i}^{(N)}\right\}$. Using (144) and (147), we have an expression of $\tilde{\nu}_{i}^{(N, \theta)}$ using $\nu_{i}^{(N)}$. Define a sequence of stopping times, $0=\tau_{i, 0}^{(N, \theta)}<\tau_{i, 1}^{(N, \theta)}<\cdots$, by

$$
\begin{aligned}
\tau_{i, 0}^{(N, \theta)}= & 0, \\
\tau_{i, k+1}^{(N, \theta)}= & \inf \left\{t>\tau_{i, k}^{(N, \theta)} \mid \nu_{i}^{(N)}\left(\left\{(s, \xi) \in\left(\tau_{i, k}^{(N, \theta)}, T\right] \times[0, \infty) \mid\right.\right.\right. \\
& \left.\left.\left.0 \leqq \xi \leqq w_{i}\left(Y_{i}^{(N, \theta)}(s-), s\right)\right\}\right)>0\right\}, k \in \mathbb{Z}_{+} .
\end{aligned}
$$

$\tau_{i, k}^{(N, \theta)}$ is the time that the particle $i$ in the flow driven stochastic ranking process jumps to the top for the $k$-th time. Put

$$
\gamma_{i}^{(N, \theta)}(t)= \begin{cases}\left(y_{i}^{(N)}, 0\right), & 0 \leqq t<\tau_{i, 1}^{(N, \theta)} \\ \left(0, \tau_{i, k}^{(N, \theta)}\right), & \tau_{i, k}^{(N, \theta)} \leqq t<\tau_{i, k+1}^{(N, \theta)}, \quad k=1,2, \ldots\end{cases}
$$


Using $\gamma_{i}^{(N, \theta)}(t)$, we have an expression

$$
\tilde{\nu}_{i}^{(N, \theta)}(t)=\int_{s \in(0, t]} \int_{\xi \in[0, \infty)} \mathbf{1}_{\xi \in\left[0, w_{i}\left(\theta\left(\gamma_{i}^{(N, \theta)}(s-), s-\right), s\right)\right)} \nu_{i}^{(N)}(d \xi d s),
$$

and substituting (34) in (31) we further have

$$
\begin{aligned}
& Y_{i}^{(N, \theta)}(t)=y_{i}^{(N)} \\
& +\frac{1}{N} \sum_{j=1}^{N} \int_{s \in(0, t]} \int_{\xi \in[0, \infty)} \mathbf{1}_{Y_{j}^{(N, \theta)}(s-)>Y_{i}^{(N, \theta)}(s-)} \\
& \times \mathbf{1}_{\xi \in\left[0, w_{j}\left(\theta\left(\gamma_{j}^{(N, \theta)}(s-), s-\right), s\right)\right)} \nu_{j}^{(N)}(d \xi d s) \\
& \quad-\int_{s \in(0, t]} \int_{\xi \in[0, \infty)} Y_{i}^{(N, \theta)}(s-) \mathbf{1}_{\xi \in\left[0, w_{i}\left(\theta\left(\gamma_{i}^{(N, \theta)}(s-), s-\right), s\right)\right)} \nu_{i}^{(N)}(d \xi d s), \\
& i=1,2, \ldots, N, \quad t \geqq 0 .
\end{aligned}
$$

This is to be compared with the (original) stochastic ranking process (3). We see that (35) is obtained from (3) by replacing $Y_{j}^{(N)}(s)$ appearing as a variable for $w_{j}$ by $\theta\left(\gamma_{j}^{(N, \theta)}(s), s\right)$, and otherwise, by $Y_{j}^{(N, \theta)}(s)$.

\subsection{Characteristic curve and distribution function.}

For each $i=1,2, \ldots, N$ and $0 \leqq t_{0} \leqq t$, define an event $J_{i}^{(N, \theta)}\left(t_{0}, t\right) \subset \Omega$ by

$$
J_{i}^{(N, \theta)}\left(t_{0}, t\right)=\left\{\omega \in \Omega \mid \tilde{\nu}_{i}^{(N, \theta)}(t)(\omega)>\tilde{\nu}_{i}^{(N, \theta)}\left(t_{0}\right)(\omega)\right\},
$$

Since $\tilde{\nu}^{(N, \theta)}$ is increasing, the complement is

$$
J_{i}^{(N, \theta)}\left(t_{0}, t\right)^{c}=\left\{\omega \in \Omega \mid \tilde{\nu}_{i}^{(N, \theta)}(t)(\omega)=\tilde{\nu}_{i}^{(N, \theta)}\left(t_{0}\right)(\omega)\right\} .
$$

On the event $J_{i}^{(N, \theta)}\left(t_{0}, t\right)^{c}$, the contribution of the last term on the right hand side of (31) to the difference $Y_{i}^{(N, \theta)}(t)-Y_{i}^{(N, \theta)}\left(t_{0}\right)$ is 0 , hence $Y_{i}^{(N, \theta)}$ is non-decreasing in the interval $\left[t_{0}, t\right]$. In other words, $J_{i}^{(N, \theta)}\left(t_{0}, t\right)$ of (36) $)$ is the event that the particle $i$ jumps to the top $y=0$ during $\left(t_{0}, t\right]$.

Define the characteristic curve for the stochastic ranking process driven by the flow $\theta$ by

$$
Y_{C}^{(N, \theta)}\left(\left(y_{0}, t_{0}\right), t\right)=y_{0}+\frac{1}{N} \sum_{j \in[1, N] ; Y_{j}^{(N, \theta)}\left(t_{0}\right) \geqq y_{0}} \mathbf{1}_{J_{j}^{(N, \theta)}\left(t_{0}, t\right)},
$$

for $\left(y_{0}, t_{0}\right) \in[0,1] \times[0, T]$ and $t \geqq t_{0}$. For example,

$$
Y_{C}^{(N, \theta)}\left(\left(1, t_{0}\right), t\right)=1, t \geqq t_{0} \geqq 0 .
$$

Since $N Y_{j}^{(N, \theta)}$ takes integer values, we can write (38) using (37) as

$$
Y_{C}^{(N, \theta)}\left(\left(y_{0}, t_{0}\right), t\right)=y_{0}+\frac{\left[N\left(1-y_{0}\right)\right]}{N}-\frac{1}{N} \sum_{j ; Y_{j}^{(N, \theta)}\left(t_{0}\right) \geqq y_{0}} \mathbf{1}_{J_{j}^{(N, \theta)}\left(t_{0}, t\right)^{c}},
$$

where $[x]$ is the largest integer not exceeding $x$.

We note the following expression corresponding to (7). 
Lemma 3 (i) For $t \geqq t_{0} \geqq 0$ and $0 \leqq y_{0} \leqq 1$, it holds that

$$
\begin{aligned}
& Y_{C}^{(N, \theta)}\left(\left(y_{0}, t_{0}\right), t\right)=y_{0}+\frac{1}{N} \sum_{j=1}^{N} \int_{s \in\left(t_{0}, t\right]} \int_{\xi \in[0, \infty)} \\
& \mathbf{1}_{Y_{j}^{(N, \theta)}(s-) \geqq Y_{C}^{(N, \theta)}\left(\left(y_{0}, t_{0}\right), s-\right)} \mathbf{1}_{\xi \in\left[0, w_{j}\left(\theta\left(\gamma_{j}^{(N, \theta)}(s-), s\right), s\right)\right)} \nu_{j}^{(N)}(d \xi d s),
\end{aligned}
$$

(ii) It holds that

$$
Y_{i}^{(N, \theta)}(t)=Y_{C}^{(N, \theta)}\left(\gamma_{i}^{(N, \theta)}(t), t\right), t \in[0, T],
$$

where $\gamma_{i}^{(N, \theta)}$ is as in (33).

Proof. Since on the event $J_{i}^{(N, \theta)}\left(t_{0}, t\right)^{c}$, the contribution of the last term on the right hand side of (31) to the difference $Y_{i}^{(N, \theta)}(t)-Y_{i}^{(N, \theta)}\left(t_{0}\right)$ disappears, $Y_{i}^{(N, \theta)}(t)-Y_{i}^{(N, \theta)}\left(t_{0}\right)$ and

$$
Y_{C}^{(N, \theta)}\left(\left(Y_{i}^{(N, \theta)}\left(t_{0}\right), t_{0}\right), t\right)-Y_{i}^{(N, \theta)}\left(t_{0}\right)
$$

should satisfy the same equation for $\left[t_{0}, t\right]$ on $J_{i}^{(N, \theta)}\left(t_{0}, t\right)^{c}$. This implies (41).

By definition (33), $J_{i}^{(N, \theta)}\left(t_{0}, t\right)^{c}$ holds if $\gamma_{i}^{(N, \theta)}(t)=\left(y_{0}, t_{0}\right)$. Hence (42) follows.

In analogy to (5), for each positive integer $N$ define a joint empirical distribution of jump rate and position of particles on $W \times[0,1]$ by

$$
\mu_{t}^{(N, \theta)}=\frac{1}{N} \sum_{i=1}^{N} \delta_{\left(w_{i}, Y_{i}^{(N, \theta)}(t)\right)} t \in[0, T] .
$$

When integrated over position, we recover the distribution $\lambda^{(N)}$ of jump rates for the (original) stochastic ranking process (23), independently of $t$ and $\theta$;

$$
\mu_{t}^{(N, \theta)}(d w \times[0,1])=\lambda^{(N)}(d w) .
$$

The initial distribution of $\mu_{t}^{(N, \theta)}$, found by substituting (31) at $t=0$ to (43), coincide with that of the original model $\mu_{0}^{(N)}$ in (6) :

$$
\mu_{0}^{(N, \theta)}=\mu_{0}^{(N)}
$$

A set of spatial distribution functions $\varphi^{(N, \theta)}$ is defined analogously to (8), by a convolution of (38) and (43);

$$
\begin{aligned}
& \varphi^{(N, \theta)}(d w, \gamma, t)=\mu_{t}^{(N, \theta)}\left(d w \times\left[Y_{C}^{(N, \theta)}(\gamma, t), 1\right]\right), \\
& \gamma=\left(y_{0}, t_{0}\right) \in[0,1] \times[0, T], t \geqq t_{0} .
\end{aligned}
$$

$\varphi^{(N, \theta)}(d w, \gamma, t)$ denotes the empirical distribution of jump rates (intensity densities) of those particles which was in a downstream of $y_{0}$ at time $t_{0}$ that have not jumped to the top in the time period $\left(t_{0}, t\right]$. For $t=t_{0}$ we have

$$
\varphi^{(N, \theta)}\left(d w,\left(y_{0}, t_{0}\right), t_{0}\right)=\mu_{t_{0}}^{(N, \theta)}\left(d w \times\left[y_{0}, 1\right]\right), \quad\left(y_{0}, t_{0}\right) \in[0,1] \times[0, T] .
$$

$\varphi^{(N, \theta)}$ is a refinement of $Y_{C}^{(N, \theta)}$ in the following sense. First, (46) and (43) imply

$$
\varphi^{(N, \theta)}(d w, \gamma, t)=\frac{1}{N} \sum_{j=1}^{N} \mathbf{1}_{Y_{j}^{(N, \theta)}(t) \geqq Y_{C}^{(N, \theta)}(\gamma, t)} \delta_{w_{j}}(d w) .
$$


Next, $Y_{j}^{(N, \theta)}(t) \geqq Y_{C}^{(N, \theta)}(\gamma, t)$ occurs if and only if the inequality holds at $t=t_{0}$ and $j$ does not jump to the top in the interval $\left(t_{0}, t\right]$. Hence (36) implies

$$
\varphi^{(N, \theta)}\left(d w,\left(y_{0}, t_{0}\right), t\right)=\frac{1}{N} \sum_{j ; Y_{j}^{(N, \theta)}\left(t_{0}\right) \geqq y_{0}} \mathbf{1}_{J_{j}^{(N, \theta)}\left(t_{0}, t\right)^{c}} \delta_{w_{j}}(d w) .
$$

Comparing (48) with (40), we have

$$
\begin{aligned}
& Y_{C}^{(N, \theta)}(\gamma, t)=y_{0}+\frac{\left[N\left(1-y_{0}\right)\right]}{N}-\varphi^{(N, \theta)}(W, \gamma, t), \\
& \gamma=\left(y_{0}, t_{0}\right) \in[0,1] \times[0, T], t \geqq t_{0}
\end{aligned}
$$

The spatial distribution function $\varphi^{(N, \theta)}(d w, \gamma, t)$ is defined for any $\gamma=\left(y_{0}, t_{0}\right) \in[0,1] \times[0, T]$ satisfying $t \geqq t_{0}$, but is particularly important when $\gamma \in \Gamma_{t}$. In fact, both for the case $\gamma=\left(y_{0}, 0\right) \in$ $\Gamma_{i}$ and the case $\gamma=\left(0, t_{0}\right) \in \Gamma_{t}$ we have

$$
\begin{aligned}
& \varphi^{(N, \theta)}\left(d w,\left(y_{0}, t_{0}\right), t\right)=\frac{1}{N} \sum_{j ; y_{j}^{(N)} \geqq y_{0}} \mathbf{1}_{J_{j}^{(N, \theta)}\left(t_{0}, t\right)^{c}} \delta_{w_{j}}(d w), \\
& \gamma=\left(y_{0}, t_{0}\right) \in \Gamma_{t}, t \in[0, T] .
\end{aligned}
$$

Note the conditions of the summation, which is non-random in (50), while is random in (48). Since $J_{j}^{(N, \theta)}\left(t_{0}, t\right)$ is independent of $\nu_{i}^{(N)}, i \neq j$, (50) implies that $\varphi^{(N, \theta)}(d w, \gamma, t)$ is an arithmetic average of independent random variables, if $\gamma \in \Gamma_{t}$. With this fact, we restrict the domain of definition to $\Delta_{T}$ of (12) in $\S 3.3$.

\subsection{Convergence of distribution function.}

\subsubsection{Statement of the theorem.}

Here we will state a strong law of large numbers for the spatial distribution function $\varphi^{(N, \theta)}$ of the flow driven stochastic ranking process, uniform in initial point $\gamma$ and time $t$.

For a bounded continuous function $h: W \rightarrow \mathbb{R}$, we put

$$
C_{h}=\sup _{w \in W}|h(w)|<\infty
$$

and use a notation

$$
\varphi^{(N, \theta)}(h, \gamma, t)=\int_{W} h(w) \varphi^{(N, \theta)}(d w, \gamma, t) .
$$

With (48) we have

$$
\varphi^{(N, \theta)}(h, \gamma, t)=\frac{1}{N} \sum_{j ; Y_{j}^{(N, \theta)}\left(t_{0}\right) \geqq y_{0}} h\left(w_{j}\right) \mathbf{1}_{J_{j}^{(N, \theta)}\left(t_{0}, t\right)^{c}}, \quad \gamma=\left(y_{0}, t_{0}\right) .
$$

As in (52) we also use a notation

$$
\varphi_{\theta}(h, \gamma, t)=\int_{W} h(w) \varphi_{\theta}(d w, \gamma, t) .
$$

for $\varphi_{\theta}$ in (17). 
Theorem 4 Assume (2), (21), (25), and (27). Then for any $p>0$ there exists a positive constant $C$ depending only on $p$ and $\delta$, (and is independent of $N, \theta$, and $h$,) such that for any bounded continuous $h: W \rightarrow \mathbb{R}$,

$$
\mathrm{E}\left[\sup _{(\gamma, t) \in \Delta_{T}}\left|\varphi^{(N, \theta)}(h, \gamma, t)-\varphi_{\theta}(h, \gamma, t)\right|^{2 p}\right] \leqq \frac{C C_{h}^{2 p}}{N^{2 p \delta}}, \quad N \in \mathbb{N},
$$

holds, where $C_{h}$ is as in (51).

Theorem 1, Theorem 4 with $h=\mathbf{1}_{W}$ and $\theta=y_{C}$, and (49) imply the following.

Corollary 5 Under the assumptions of Theorem 4, for any $p>0$ there exists a positive constant $C$ depending only on $p$ and $\delta$, such that

$$
\mathrm{E}\left[\sup _{(\gamma, t) \in \Delta_{T}}\left|Y_{C}^{\left(N, y_{C}\right)}(\gamma, t)-y_{C}(\gamma, t)\right|^{2 p}\right] \leqq \frac{C}{N^{2 p \delta}}, N \in \mathbb{N} .
$$

Among $\theta \in \Theta, \theta=y_{C}$ is the only flow that satisfies (56).

As in the proof of the main theorem Theorem 2, Theorem 4 implies convergence of joint empirical

distribution $\mu_{t}^{(N, \theta)}$ to $\mu_{y_{C}, t}$, a deterministic distribution determined by $\varphi_{y_{C}}(h, \gamma, t)$. For $\theta \neq y_{C}$, the stochastic ranking process driven by the flow $\theta$ converges to a limit with deterministic distribution, but the resulting trajectories of particles are different from the given flow $\theta$, due to the uniqueness result of Theorem 1 .

A proof of Theorem 4 is composed of 2 parts. In $\S 3.3 .2$ we prove that $\varphi^{(N, \theta)}-\mathrm{E}\left[\varphi^{(N, \theta)}\right]$ converges to 0 , using a strong uniform law of large numbers in [12]. In $\S 3.3 .3$ we prove that $\mathrm{E}\left[\varphi^{(N, \theta)}\right]$ converges to $\varphi_{\theta}$, using the estimates in [10]. Relevant results of [10] are summarized in $\S$ A for convenience.

\subsubsection{Strong uniform law of large numbers.}

Here we will prove the following.

Theorem 6 Assume (2), (21), (25), and 27). Then for any $p$ and $\delta$ satisfying

$$
p>0, \quad 0<\delta<\frac{1}{2},
$$

there exists a positive constant $C$ (independent of $N, \theta$, and $h$,) such that for any bounded continuous $h: W \rightarrow \mathbb{R}$

$$
\mathrm{E}\left[\sup _{(\gamma, t) \in \Delta_{T}}\left|\varphi^{(N, \theta)}(h, \gamma, t)-\mathrm{E}\left[\varphi^{(N, \theta)}(h, \gamma, t)\right]\right|^{2 p}\right] \leqq \frac{C C_{h}^{2 p}}{N^{2 p \delta}}, N \in \mathbb{N},
$$

where $C_{h}$ is as in (51).

Proof. Put $h_{ \pm}=( \pm h) \vee 0$, so that $h=h_{+}-h_{-}$decomposes the function $h$ to positive and negative parts. Applying (48), (12), (11), and triangular inequality in the form

$$
(a+b)^{q} \leqq\left(2^{q-1} \vee 1\right)\left(a^{q}+b^{q}\right), a \geqq 0, b \geqq 0, q>0,
$$


we have, with $\gamma=\left(y_{0}, t_{0}\right) \in \Gamma_{t}$,

$$
\begin{aligned}
& \mathrm{E}\left[\sup _{\left(\left(y_{0}, t_{0}\right), t\right) \in \Delta_{T}}\left|\varphi^{(N, \theta)}(h, \gamma, t)-\mathrm{E}\left[\varphi^{(N, \theta)}(h, \gamma, t)\right]\right|^{q}\right] \\
& =\mathrm{E}\left[\sup _{\left(\left(y_{0}, t_{0}\right), t\right)} \mid \frac{1}{N} \sum_{Y_{j}^{(N, \theta)}\left(t_{0}\right) \geqq y_{0}}\left(h_{+}\left(w_{j}\right)-h_{-}\left(w_{j}\right)\right)\right. \\
& \left.\times\left.\left(\mathbf{1}_{J_{j}^{(N, \theta)}\left(t_{0}, t\right)^{c}}-\mathrm{E}\left[\mathbf{1}_{J_{j}^{(N, \theta)}\left(t_{0}, t\right)^{c}}\right]\right)\right|^{q}\right] \\
& \leqq\left(2^{q-1} \vee 1\right) \mathrm{E}\left[\sup _{\left(\left(y_{0}, t_{0}\right), t\right)} \mid \frac{1}{N} \sum_{Y_{j}^{(N, \theta)}\left(t_{0}\right) \geqq y_{0}} h_{+}\left(w_{j}\right)\right. \\
& \left.\times\left.\left(\mathbf{1}_{J_{j}^{(N, \theta)}\left(t_{0}, t\right)^{c}}-\mathrm{E}\left[\mathbf{1}_{J_{j}^{(N, \theta)}\left(t_{0}, t\right)^{c}}\right]\right)\right|^{q}\right] \\
& +\left(2^{q-1} \vee 1\right) \mathrm{E}\left[\sup _{\left(\left(y_{0}, t_{0}\right), t\right)} \mid \frac{1}{N} \sum_{Y_{j}^{(N, \theta)}\left(t_{0}\right) \geqq y_{0}} h_{-}\left(w_{j}\right)\right. \\
& \left.\times\left.\left(\mathbf{1}_{J_{j}^{(N, \theta)}\left(t_{0}, t\right)^{c}}-\mathrm{E}\left[\mathbf{1}_{J_{j}^{(N, \theta)}\left(t_{0}, t\right)^{c}}\right]\right)\right|^{q}\right] \\
& \leqq\left(2^{q-1} \vee 1\right)\left(R_{q, 1,+}^{(N)}+R_{q, 1,-}^{(N)}+R_{q, 2,+}^{(N)}+R_{q, 2,-}^{(N)}\right),
\end{aligned}
$$

where

$$
R_{q, 1, \pm}^{(N)}=\mathrm{E}\left[\sup _{t \in[0, T]} \sup _{0 \leqq y_{0}<1}\left|\frac{1}{N} \sum_{i ; y_{i}^{(N)} \geqq y_{0}} h_{ \pm}\left(w_{i}\right)\left(\mathbf{1}_{J_{i}^{(N, \theta)}(0, t)^{c}}-\mathrm{E}\left[\mathbf{1}_{J_{i}^{(N, \theta)}(0, t)^{c}}\right]\right)\right|^{q}\right]
$$

and

$$
R_{q, 2, \pm}^{(N)}=\mathrm{E}\left[\sup _{t \in[0, T]} \sup _{0 \leqq t_{0}<t}\left|\frac{1}{N} \sum_{i=1}^{N} h_{ \pm}\left(w_{i}\right)\left(\mathbf{1}_{J_{i}^{(N, \theta)}\left(t_{0}, t\right)^{c}}-\mathrm{E}\left[\mathbf{1}_{J_{i}^{(N, \theta)}\left(t_{0}, t\right)^{c}}\right]\right)\right|^{q}\right] .
$$

To bound (60) and (61), we refer to the last theorem in [12, §2]. We reproduce the theorem in a specific form of

$$
Z_{i}^{(N)}(s, t)=a_{i}^{(N)} \mathbf{1}_{\nu_{i}^{(N)}(t)>\nu_{i}^{(N)}(s)}
$$

in place of $Z_{i}^{(N)}(s, t)$ in the reference.

Proposition 7 ([12, Theorem 3]) Let $T>0$, and for each $N \in \mathbb{N}$, let $\nu_{i}^{(N)}, i=1 \ldots, N$, be a sequence of independent random variables taking values in a space of non-negative valued non-decreasing right continuous functions on $[0, T]$ with left limit. Let $r>0$, and for $N \in \mathbb{N}$, let $M^{(N)}>0$ and let $a_{i}^{(N)}$ and $w_{i}^{(N)}, i=1, \ldots, N$, be non-negative sequences. Assume that $a_{i}^{(N)} \leqq M^{(N)}, i=1, \ldots, N$, and that

$$
\begin{aligned}
& \left|\mathrm{P}\left[\nu_{i}^{(N)}\left(t_{2}\right)>\nu_{i}^{(N)}\left(t_{1}\right)\right]-\mathrm{P}\left[\nu_{i}^{(N)}\left(s_{2}\right)>\nu_{i}^{(N)}\left(s_{1}\right)\right]\right| \\
& \leqq w_{i}^{(N)}\left(\left|t_{1}-s_{1}\right|^{r}+\left|t_{2}-s_{2}\right|^{r}\right),
\end{aligned}
$$

for $0 \leqq t_{1} \leqq t_{2} \leqq T$ and $0 \leqq s_{1} \leqq s_{2} \leqq T$. Then for any $\delta \in\left(0, \frac{1}{2}\right)$ and $p>0$,

$$
\begin{aligned}
& \mathrm{E}\left[\sup _{t_{2} \in[0, T]} \sup _{0 \leqq t_{1}<t_{2}}\left|\frac{1}{N} \sum_{i=1}^{N} a_{i}^{(N)}\left(\mathbf{1}_{\nu_{i}^{(N)}\left(t_{2}\right)>\nu_{i}^{(N)}\left(t_{1}\right)}-\mathrm{P}\left[\nu_{i}^{(N)}\left(t_{2}\right)>\nu_{i}^{(N)}\left(t_{1}\right)\right]\right)\right|^{p}\right]^{1 / p} \\
& \leqq \frac{M^{(N)}}{N^{\delta}} 2^{1-1 / q}\left(C_{q}^{q}\left(2 T\left(\bar{w}^{(N)}\right)^{1 / r}+1\right)+2^{2 q}\right)^{1 / q} \\
& N=N_{0}, N_{0}+1, \ldots,
\end{aligned}
$$


where $q=q(p, \delta)=3 \vee \frac{r+1}{r} \frac{2 \delta}{1-2 \delta} \vee p, N_{0}$ is the smallest integer satisfying $N_{0}^{r q /(2 r q+2 r+2)} \geqq 2$, $\bar{w}^{(N)}=\frac{1}{N} \sum_{i=1}^{N} w_{i}^{(N)}$, and $C_{q}=\left(\frac{1}{2}(4 k)^{q}+\frac{2 k}{2 k-q}(8 k)^{q}\right)^{1 / q}$ with $k$ the smallest integer greater than $\frac{1}{2} q$, (In particular, $q$ and $N_{0}$ are independent of $N, M^{(N)}$, and $\bar{w}^{(N)}$.)

To bound $R_{q, 2, \pm}^{(N)}$ in (61), we apply Proposition 7 with $\nu_{i}^{(N)}=\tilde{\nu}_{i}^{(N, \theta)}$ and $a_{i}^{(N)}=h_{ \pm}\left(w_{i}\right)$. Note that (152) and (16) with $w=w_{i}$ and $z=y_{i}^{(N)}$, and (11) imply

$$
\begin{aligned}
& 0 \leqq-\frac{\partial}{\partial t} \mathrm{P}\left[\tilde{\nu}_{i}^{(N, \theta)}(t)=\tilde{\nu}_{i}^{(N, \theta)}(s)\right] \leqq\left\|\tilde{w}_{\theta, w_{i}, y_{i}^{(N)}}\right\| \leqq\left\|w_{i}\right\|_{\mathrm{T}}, \\
& 0 \leqq \frac{\partial}{\partial s} \mathrm{P}\left[\tilde{\nu}_{i}^{(N, \theta)}(t)=\tilde{\nu}_{i}^{(N, \theta)}(s)\right] \leqq\left\|\tilde{w}_{\theta, w_{i}, y_{i}^{(N)}}\right\| \leqq\left\|w_{i}\right\|_{\mathrm{T}} .
\end{aligned}
$$

Recall (36) and (37). Comparing the left hand side of (62) with the right hand side of (61) with $q=2 p$, we see that we can apply Proposition 7 to $R_{2 p, 2, \pm}^{(N)}$ with

$$
M^{(N)}=C_{h}, \quad r=1, \quad w_{i}^{(N)}=\left\|w_{i}\right\|_{\mathrm{T}} .
$$

Proposition 7 then implies that for any $\delta \in\left(0, \frac{1}{2}\right)$ and $p>0$,

$$
R_{2 p, 2, \pm}^{(N)} \leqq \frac{C_{h}^{2 p}}{N^{2 p \delta}} 2^{2 p-1}\left(C_{q}^{q}\left(2 T \bar{w}^{(N)}+1\right)+2^{2 q}\right)^{2 p / q}, \quad N>2^{2+(4 / q)},
$$

where

$$
\begin{aligned}
& q=q(p, \delta)=3 \vee \frac{4 \delta}{1-2 \delta} \vee(2 p), \\
& \bar{w}^{(N)}=\frac{1}{N} \sum_{i=1}^{N}\left\|w_{i}\right\|_{\mathrm{T}}=\int_{W}\|w\|_{\mathrm{T}} \lambda^{(N)}(d w),
\end{aligned}
$$

and $C_{q}$ is a positive constant depending only on $q$.

Combining (64) $\mathrm{C}(\underline{65})$, and (27), we see that there exists a positive constant $C_{p, \delta}$ independent of $N, \theta$, and $h$, such that

$$
R_{2 p, 2, \pm}^{(N)} \leqq \frac{C_{p, \delta} C_{h}^{2 p}}{N^{2 p \delta}}, N \in \mathbb{N}
$$

To bound $R_{q, 1, \pm}^{(N)}$ in (60), we first note that

$$
\begin{aligned}
& R_{q, 1, \pm}^{(N)} \\
& \leqq \sum_{j=1}^{N} \mathrm{E}\left[\sup _{t \in[0, T]}\left|\frac{1}{N} \sum_{i ; N y_{i}^{(N)} \geqq j-1} h_{ \pm}\left(w_{i}\right)\left(\mathbf{1}_{J_{i}^{(N, \theta)}(0, t)^{c}}-\mathrm{E}\left[\mathbf{1}_{J_{i}^{(N, \theta)}(0, t)^{c}}\right]\right)\right|^{q}\right] \\
& \leqq \sum_{j=1}^{N} \mathrm{E}\left[\sup _{t \in[0, T]} \sup _{0 \leqq t_{0}<t}\left|\frac{1}{N} \sum_{i ; N y_{i}^{(N)} \geqq j-1} h_{ \pm}\left(w_{i}\right)\left(\mathbf{1}_{J_{i}^{(N, \theta)}\left(t_{0}, t\right)^{c}}-\mathrm{E}\left[\mathbf{1}_{J_{i}^{(N, \theta)}\left(t_{0}, t\right)^{c}}\right]\right)\right|^{q}\right] .
\end{aligned}
$$

Comparing (67) with (61), we see that we can apply Proposition 7 with $\nu_{i}^{(N)}=\tilde{\nu}_{i}^{(N, \theta)}$ and

$$
a_{i}^{(N)}=h_{ \pm}\left(w_{i}\right) \mathbf{1}_{N y_{i}^{(N)} \geqq j-1},
$$


and (64), to the $j$-th term of the summation in the right hand side of (67), in a similar way as we $\operatorname{did}$ to $R_{2 p, 2, \pm}^{(N)}$. Using monotonicity of $L^{p}$ norms with respect to $p$ before applying Proposition 7 . we have, for $q_{0} \geqq 2 p$ and $\delta^{\prime} \in\left(0, \frac{1}{2}\right)$,

$$
R_{2 p, 1, \pm} \leqq R_{q_{0}, 1, \pm}^{2 p / q_{0}} \leqq \frac{\left(C_{q_{0} / 2, \delta^{\prime}} C_{h}^{q_{0}}\right)^{2 p / q_{0}}}{N^{2 p \delta^{\prime}-2 p / q_{0}}}, N \in \mathbb{N}
$$

in place of (66). (The extra factor $N$ compared to (66) is from the summation with respect to $j$ in (67).) Now choose $\delta^{\prime}$ and $q_{0}$ to satisfy $\delta<\delta^{\prime}<\frac{1}{2}$ and $0<\frac{1}{q_{0}}<\delta^{\prime}-\delta$ to find

$$
R_{2 p, 1, \pm} \leqq R_{q_{0}, 1, \pm}^{2 p / q_{0}} \leqq \frac{\left(C_{q_{0} / 2, \delta^{\prime}} C_{h}^{q_{0}}\right)^{2 p / q_{0}}}{N^{2 p \delta}}, N \in \mathbb{N}
$$

Theorem 6 finally follows from (59), (68), and (66).

\subsubsection{Convergence of expectation.}

Here we complete a proof of Theorem 4 .

Lemma 8 Assume 27). Then, if $h: W \rightarrow \mathbb{R}$ is bounded and continuous,

$$
\lim _{N \rightarrow \infty} \int_{W} h(w)\|w\|_{\mathrm{T}} \lambda^{(N)}(d w)=\int_{W} h(w)\|w\|_{\mathrm{T}} \lambda(d w) .
$$

Proof. Note first that for $M>0$

$$
a=(a \wedge M)+(a-M)_{+}
$$

holds. Since $w \mapsto\|w\|_{\mathrm{T}} \wedge M$ is bounded and continuous, (27) (weak convergence) implies

$$
\lim _{N \rightarrow \infty} \int_{W}\|w\|_{\mathrm{T}} \wedge M \lambda^{(N)}(d w)=\int_{W}\|w\|_{\mathrm{T}} \wedge M \lambda(d w)
$$

which, with convergence of expectation in (27), further implies

$$
\lim _{N \rightarrow \infty} \int_{W}\left(\|w\|_{\mathrm{T}}-M\right)_{+} \lambda^{(N)}(d w)=\int_{W}\left(\|w\|_{\mathrm{T}}-M\right)_{+} \lambda(d w) .
$$

On the other hand, dominated convergence theorem, $0 \leqq\left(\|w\|_{\mathrm{T}}-M\right)_{+} \leqq\|w\|_{\mathrm{T}}$, and (25) imply

$$
\lim _{M \rightarrow \infty} \int_{W}\left(\|w\|_{\mathrm{T}}-M\right)_{+} \lambda(d w)=0
$$

Hence, for any $\epsilon>0$ there exists $M>0$ such that $\int_{W}\left(\|w\|_{\mathrm{T}}-M\right)_{+} \lambda(d w)<\epsilon$. This and (172) further imply that there exists $N_{0}>0$ such that

$$
\int_{W}\left(\|w\|_{\mathrm{T}}-M\right)_{+} \lambda^{(N)}(d w)<2 \epsilon, \quad N \geqq N_{0} .
$$


Put $C=\sup _{w \in W}|h(w)|<\infty$. Then

$$
\begin{aligned}
& \left|\int_{W} h(w)\|w\|_{\mathrm{T}} \lambda^{(N)}(d w)-\int_{W} h(w)\|w\|_{\mathrm{T}} \lambda(d w)\right| \\
& \leqq\left|\int_{W}\|w\|_{\mathrm{T}} \wedge M \lambda^{(N)}(d w)-\int_{W}\|w\|_{\mathrm{T}} \wedge M \lambda(d w)\right| \\
& \quad+C \int_{W}\left(\|w\|_{\mathrm{T}}-M\right)_{+} \lambda^{(N)}(d w)+C \int_{W}\left(\|w\|_{\mathrm{T}}-M\right)_{+} \lambda(d w) \\
& \leqq\left|\int_{W}\|w\|_{\mathrm{T}} \wedge M \lambda^{(N)}(d w)-\int_{W}\|w\|_{\mathrm{T}} \wedge M \lambda(d w)\right|+3 C \epsilon .
\end{aligned}
$$

This and (71) imply

$$
\varlimsup_{N \rightarrow \infty}\left|\int_{W} h(w)\|w\|_{\mathrm{T}} \lambda^{(N)}(d w)-\int_{W} h(w)\|w\|_{\mathrm{T}} \lambda(d w)\right| \leqq 3 C \epsilon .
$$

Since the left hand side is independent of $N, M$, and $\epsilon$, it must be 0 .

Proof of Theorem 4. Let $h: W \rightarrow \mathbb{R}$ be a bounded continuous function, and put $C_{h}=\sup _{w \in W}|h(w)|$ as in (51). Theorem 6 implies that to prove Theorem 4, it suffices to prove

$$
\sup _{(\gamma, t) \in \Delta_{T}}\left|\mathrm{E}\left[\varphi^{(N, \theta)}(h, \gamma, t)\right]-\varphi_{\theta}(h, \gamma, t)\right| \leqq \frac{C C_{h}}{N^{\delta}}, N \in \mathbb{N}
$$

for $C$ depending only on $p$ and $\delta$ (independent of $N, \theta$, and $h$ ).

Comparing the definition of $\tilde{\nu}_{i}^{(N, \theta)}$ given above (31) with that of $\tilde{\nu}_{\theta, w, z}$ given below (17), we see that $\tilde{\nu}_{i}^{(N, \theta)}$ and $\tilde{\nu}_{\theta, w_{i}, y_{i}^{(N)}}$ have identical distribution. Therefore, using (37) and (6) in (53)), we have, for $t \geqq t_{0}$,

$$
\mathrm{E}\left[\varphi^{(N, \theta)}\left(h,\left(y_{0}, t_{0}\right), t\right)\right]=\int_{W \times\left[y_{0}, 1\right]} h(w) \mathrm{P}\left[\tilde{\nu}_{\theta, w, z}(t)=\tilde{\nu}_{\theta, w, z}\left(t_{0}\right)\right] \mu_{0}^{(N)}(d w \times d z) .
$$

Let $\gamma=\left(y_{0}, t_{0}\right) \in \Gamma$ and $(\gamma, t) \in \Delta_{T}$. (74) and (17) imply

$$
\begin{aligned}
& \mathrm{E}\left[\varphi^{(N, \theta)}(h, \gamma, t)\right]-\varphi_{\theta}(h, \gamma, t) \\
& =\int_{W \times\left[y_{0}, 1\right]} h(w) \mathrm{P}\left[\tilde{\nu}_{\theta, w, z}(t)=\tilde{\nu}_{\theta, w, z}\left(t_{0}\right)\right]\left(\mu_{0}^{(N)}(d w \times d z)-\mu_{0}(d w \times d z)\right) .
\end{aligned}
$$

Hence,

$$
\begin{aligned}
& \sup _{(\gamma, t) \in \Delta_{T}}\left|\mathrm{E}\left[\varphi^{(N, \theta)}(h, \gamma, t)\right]-\varphi_{\theta}(h, \gamma, t)\right| \\
& \leqq \sup _{\left(\left(y_{0}, t_{0}\right), t\right) \in \Delta_{T}} \mid \int_{W \times\left[y_{0}, 1\right]} \tilde{h}_{t_{0}, t}(w, z)\left(\mu_{0}^{(N)}(d w \times d z)-\mu_{0}(d w \times d z) \mid,\right.
\end{aligned}
$$

where

$$
\tilde{h}_{t_{0}, t}(w, z)=h(w) \mathrm{P}\left[\tilde{\nu}_{\theta, w, z}(t)=\tilde{\nu}_{\theta, w, z}\left(t_{0}\right)\right] .
$$

Choose the set $H$ in (21) as the set of the functions $\tilde{h}_{t_{0}, t}$ in (76):

$$
H=\left\{\tilde{h}_{t_{0}, t}: W \times[0,1] \rightarrow \mathbb{R} \mid 0 \leqq t_{0} \leqq t \leqq T\right\} .
$$


Uniform boundedness of the functions in $H$ is obvious. If we prove that $H$ is also equicontinuous, then by the assumption of Theorem 4 the consequence of (21) holds, which implies

$$
\sup _{\substack{y_{0} \in[0,1] \\ N \in \mathbb{N} .}} \sup _{\substack{N \leq t_{0} \leqq t \leqq T}} \mid \int_{W \times\left[y_{0}, 1\right]} \tilde{h}_{t_{0}, t}(w, z)\left(\mu_{0}^{(N)}(d w \times d z)-\mu_{0}(d w \times d z) \mid \leqq \frac{C C_{h}}{N^{\delta}},\right.
$$

Applying this estimate to (75), we have (73), which proves Theorem 4 .

We are left with proving equicontinuity of $H$.

First, for $(w, z) \in W \times[0,1]$ and $\tilde{w}_{\theta, w, z}$ as in (16) (i.e., the 'intensity density' for $\tilde{\nu}_{\theta, w, z}$ ), and $0 \leqq s \leqq t \leqq T$, put

$$
\Omega_{\theta, w, z}(s, t)=\int_{s}^{t} \tilde{w}_{\theta, w, z}(s, u) d u \text { and } \tilde{\Omega}_{w}(s, t)=\int_{s}^{t} w(1, u) d u .
$$

Then (2) and a mean value theorem imply

$$
w(1, t)-C_{W} \leqq \tilde{w}_{\theta, w, z}(s, t) \leqq w(1, t)+C_{W},
$$

and

$$
e^{-\tilde{\Omega}_{w}(s, t)-C_{W}(t-s)} \leqq e^{-\Omega_{\theta, w, z}(s, t)} \leqq e^{-\tilde{\Omega}_{w}(s, t)+C_{W}(t-s)} .
$$

Note also an elementary formula in [10, (53)]

$$
\int_{0 \leqq u_{1} \leqq u_{2} \leqq \ldots \leqq u_{k} \leqq s} \prod_{i=1}^{k} f\left(u_{i}\right) d u_{1} d u_{2} \ldots d u_{k}=\frac{1}{k !}\left(\int_{0}^{s} f(v) d v\right)^{k},
$$

valid for any integrable function $f: \mathbb{R} \rightarrow \mathbb{R}, s \geqq 0$, and $k=1,2, \ldots$,

A proof of equicontinuity of $H$ now goes in a similar way as that of [10, Lemma 12]. Applying (151) to (76), we have

$$
\begin{aligned}
\tilde{h}_{t_{0}, t}(w, z)= & h(w) \sum_{k \geqq 0} \int_{0=: u_{k}<u_{k-1}<u_{k-2}<\cdots<u_{1}<u_{0} \leqq t_{0}} \\
& \times e^{-\sum_{i=0}^{k-1} \Omega_{\theta, w, z}\left(u_{i+1}, u_{i}\right)-\Omega_{\theta, w, z}\left(u_{0}, t\right)}\left(\prod_{i=0}^{k-1} \tilde{w}_{\theta, w, z}\left(u_{i+1}, u_{i}\right) d u_{i}\right) .
\end{aligned}
$$

Using (79), (80), and (78) to (82), while noting that (16) implies that $\tilde{w}_{\theta, w, z}(s, t)$ and $\Omega_{\theta, w, z}(s, t)$ is independent of $z$ if $s>0$, we have

$$
\left|\tilde{h}_{t_{0}, t}\left(w, z^{\prime}\right)-\tilde{h}_{t_{0}, t}(w, z)\right| \leqq I_{11}\left(z, z^{\prime}\right)+I_{12}\left(z, z^{\prime}\right)
$$

where

$$
\begin{aligned}
I_{11}\left(z, z^{\prime}\right)= & C_{h} e^{-\tilde{\Omega}_{w}(0, t)+C_{W} t} \sum_{k \geqq 1} \int_{0=: u_{k}<u_{k-1}<u_{k-2}<\cdots<u_{1}<u_{0} \leqq t_{0}} \\
& \times\left(\prod_{i=0}^{k-2}\left(w\left(1, u_{i}\right)+C_{W}\right) d u_{i}\right) \\
& \times\left|\tilde{w}_{\theta, w, z^{\prime}}\left(0, u_{k-1}\right)-\tilde{w}_{\theta, w, z}\left(0, u_{k-1}\right)\right| d u_{k-1},
\end{aligned}
$$


and

$$
\begin{aligned}
I_{12}\left(z, z^{\prime}\right)= & C_{h} \sum_{k \geqq 0} \int_{0=: u_{k}<u_{k-1}<u_{k-2}<\cdots<u_{1}<u_{0} \leqq t_{0}} e^{-\tilde{\Omega}_{w}\left(u_{k-1}, t\right)+C_{W}\left(t-u_{k-1}\right)} \\
& \times\left(\prod_{i=0}^{k-1}\left(w\left(1, u_{i}\right)+C_{W}\right) d u_{i}\right) \\
& \times\left|e^{-\Omega_{\theta, w, z^{\prime}}\left(0, u_{k-1}\right)}-e^{-\Omega_{\theta, w, z}\left(0, u_{k-1}\right)}\right| d u_{k-1} .
\end{aligned}
$$

Using (16), (22), (81), and (78), we have

$$
\begin{aligned}
I_{11}\left(z, z^{\prime}\right) & \leqq C_{h} C_{W} e^{-\tilde{\Omega}_{w}\left(t_{0}, t\right)+C_{W}\left(t+t_{0}\right)} \int_{0}^{t_{0}}\left|\theta\left(\left(z^{\prime}, 0\right), v\right)-\theta((z, 0), v)\right| d v \\
& \leqq C_{h} C_{W} e^{2 C_{W} T} \int_{0}^{T}\left|\theta\left(\left(z^{\prime}, 0\right), v\right)-\theta((z, 0), v)\right| d v .
\end{aligned}
$$

Using in addition

$$
\begin{aligned}
& \left|e^{-x^{\prime}}-e^{-x}\right|=e^{-\left(x^{\prime} \wedge x\right)}-e^{-\left(x^{\prime} \vee x\right)}=e^{-\left(x^{\prime} \wedge x\right)}\left(1-e^{-\left|x^{\prime}-x\right|}\right) \\
& \leqq e^{-\left(x^{\prime} \wedge x\right)}\left|x^{\prime}-x\right| \leqq e^{-x} e^{\left|x^{\prime}-x\right|}\left|x^{\prime}-x\right|
\end{aligned}
$$

which follows from $\left|x^{\prime}-x\right|=\left(x^{\prime} \vee x\right)-\left(x^{\prime} \wedge x\right) \geqq x-\left(x \wedge x^{\prime}\right)$, we similarly have

$$
\begin{aligned}
I_{12}\left(z, z^{\prime}\right) \leqq & C_{h} C_{W} e^{-\tilde{\Omega}_{w}\left(t_{0}, t\right)+C_{W}\left(t+t_{0}\right)} \int_{0}^{t_{0}}\left|\theta\left(\left(z^{\prime}, 0\right), v\right)-\theta((z, 0), v)\right| d v \\
& \times e^{C_{W} \int_{0}^{t_{0}}\left|\theta\left(\left(z^{\prime}, 0\right), v\right)-\theta((z, 0), v)\right| d v} \\
\leqq & C_{h} C_{W} e^{2 C_{W} T} \int_{0}^{T}\left|\theta\left(\left(z^{\prime}, 0\right), v\right)-\theta((z, 0), v)\right| d v \\
& \times e^{C_{W} \int_{0}^{T}\left|\theta\left(\left(z^{\prime}, 0\right), v\right)-\theta((z, 0), v)\right| d v} .
\end{aligned}
$$

Since the right hand sides of the bounds for $I_{11}$ and $I_{12}$ are uniform in $t_{0}$ and $t$, these prove equicontinuity in the variable $z \in[0,1]$ of functions $\tilde{h}_{t_{0}, t}(w, z)$ in $H$.

In a similar way as the proof of equicontinuity with respect to $z$, we have

$$
\left|\tilde{h}_{t_{0}, t}\left(w^{\prime}, z\right)-\tilde{h}_{t_{0}, t}(w, z)\right| \leqq I_{21}\left(w, w^{\prime}\right)+I_{22}\left(w, w^{\prime}\right),
$$

where

$$
\begin{aligned}
I_{21}\left(w, w^{\prime}\right)= & C_{h} \sum_{k \geqq 0} \int_{0=: u_{k}<u_{k-1}<u_{k-2}<\cdots<u_{1}<u_{0} \leqq t_{0}} \\
& \times\left|e^{-X\left(w^{\prime}\right)}-e^{-X(w)}\right|\left(\prod_{i=0}^{k-1}\left(w^{\prime}\left(1, u_{i}\right)+C_{W}\right) d u_{i}\right) d u_{k-1},
\end{aligned}
$$

with

$$
X(w)=\sum_{i=0}^{k-1} \Omega_{\theta, w, z}\left(u_{i+1}, u_{i}\right)+\Omega_{\theta, w, z}\left(u_{0}, t\right)
$$

and

$$
\begin{aligned}
I_{22}\left(w, w^{\prime}\right)= & C_{h} e^{-\tilde{\Omega}_{w}(0, t)+C_{W} t} \sum_{k \geqq 1} \int_{0=: u_{k}<u_{k-1}<u_{k-2}<\cdots<u_{1}<u_{0} \leqq t_{0}} \\
& \times\left|\prod_{i=0}^{k-1} \tilde{w}_{\theta, w^{\prime}, z}\left(u_{i+1}, u_{i}\right)-\prod_{i=0}^{k-1} \tilde{w}_{\theta, w, z}\left(u_{i+1}, u_{i}\right)\right| \prod_{i=0}^{k-1} d u_{i} .
\end{aligned}
$$


Note that (78) with (11) implies

$$
\left|\Omega_{\theta, w^{\prime}, z}(u, v)-\Omega_{\theta, w, z}(u, v)\right| \leqq\left\|w^{\prime}-w\right\|_{\mathrm{T}}(v-u), \quad 0 \leqq u \leqq T .
$$

Using this, (83), and (81) in $I_{21}\left(w, w^{\prime}\right)$, we further have

$$
\begin{aligned}
I_{21}\left(w, w^{\prime}\right) & \leqq C_{h} e^{-\tilde{\Omega}_{w}(0, t)+C_{W} t} e^{\left\|w^{\prime}-w\right\|_{\mathrm{T}} t}\left\|w^{\prime}-w\right\|_{\mathrm{T}} t e^{\tilde{\Omega}_{w^{\prime}}\left(0, t_{0}\right)+C_{W} t_{0}} \\
& \leqq C_{h} e^{2 C_{W} T} e^{\left\|w^{\prime}-w\right\|_{\mathrm{T}} T}\left\|w^{\prime}-w\right\|_{\mathrm{T}} T .
\end{aligned}
$$

With a similar argument, we also have

$$
\begin{aligned}
I_{22}\left(w, w^{\prime}\right) \leqq & C_{h} e^{-\tilde{\Omega}_{w}(0, t)+C_{W} t} \sum_{k \geqq 1} \sum_{j=0}^{k-1} \int_{0=: u_{k}<u_{k-1}<u_{k-2}<\cdots<u_{1}<u_{0} \leqq t_{0}} \\
& \times\left(\prod_{i=0}^{j-1} \tilde{w}_{\theta, w^{\prime}, z}\left(u_{i+1}, u_{i}\right)\right)\left|\tilde{w}_{\theta, w^{\prime}, z}\left(u_{j+1}, u_{j}\right)-\tilde{w}_{\theta, w, z}\left(u_{j+1}, u_{j}\right)\right| \\
& \times\left(\prod_{i=j+1}^{k-1} \tilde{w}_{\theta, w, z}\left(u_{i+1}, u_{i}\right)\right) \prod_{i=0}^{k-1} d u_{i} \\
\leqq & C_{h} e^{-\tilde{\Omega}_{w}(0, t)+C_{W} t}\left\|w^{\prime}-w\right\|_{\mathrm{T}} \int_{0}^{t_{0}} e^{\tilde{\Omega}_{w}(0, v)+C_{W} v} e^{\tilde{\Omega}_{w^{\prime}}\left(v, t_{0}\right)+C_{W}\left(t_{0}-v\right)} d v \\
\leqq & C_{h} e^{-\tilde{\Omega}_{w}(0, t)+C_{W} t}\left\|w^{\prime}-w\right\|_{\mathrm{T}} \\
& \times\left(e^{\tilde{\Omega}_{w}\left(0, t_{0}\right)} t_{0}+\int_{0}^{t_{0}} e^{\tilde{\Omega}_{w^{\prime}}(0, v)}\left|e^{\tilde{\Omega}_{w^{\prime}}\left(v, t_{0}\right)}-e^{\tilde{\Omega}_{w}\left(v, t_{0}\right)}\right| d v\right) \\
\leqq & C_{h} e^{2 C_{W} T}\left\|w^{\prime}-w\right\|_{\mathrm{T}} T\left(1+e^{\left\|w^{\prime}-w\right\|_{\mathrm{T}} T}\left\|w^{\prime}-w\right\|_{\mathrm{T}} T\right) .
\end{aligned}
$$

Since the right hand sides of the bounds for $I_{21}$ and $I_{22}$ are uniform in $t_{0}$ and $t$, these prove equicontinuity of $H$ in $w \in W$.

This completes a proof of equicontinuity of $H$, hence a proof of Theorem 4 .

\section{Hierarchy of multi time Gronwall inequality.}

The following is a simple form of Gronwall's inequality.

Theorem 9 Let $T$ be a positive constant, and $a$ and $c$ be non-negative constants. If $x:[0, T] \rightarrow \mathbb{R}$ is an integrable function, satisfying

$$
x(t) \leqq a+c \int_{0}^{t} x(s) d s, t \in[0, T],
$$

then

$$
x(t) \leqq a e^{c t}, t \in[0, T]
$$

holds.

The following is a generalization of Theorem 9 to functions of more than 1 variables, where the case $q=1$ is Theorem 9 , 
Theorem 10 Let $T$ be a positive constant, $q$ a positive integer, and a and c non-negative constants. If $x:[0, T]^{q} \rightarrow \mathbb{R}$ is an integrable function of $q$ variables, satisfying

$$
\begin{aligned}
x\left(t_{1}, \ldots, t_{q}\right) \leqq & a e^{c\left(t_{1}+\cdots+t_{q}\right)} \frac{1}{q} \sum_{i=1}^{q} e^{-c t_{i}} \\
& +\frac{c}{q} \sum_{i=1}^{q} \int_{0}^{t_{i}}\left(\left.x\left(t_{1}, \ldots, t_{q}\right)\right|_{t_{i}=u}\right) d u, \quad\left(t_{1}, \ldots, t_{q}\right) \in[0, T]^{q},
\end{aligned}
$$

then

$$
x\left(t_{1}, \ldots, t_{q}\right) \leqq a e^{c\left(t_{1}+\cdots+t_{q}\right)}, \quad\left(t_{1}, \ldots, t_{q}\right) \in[0, T]^{q},
$$

holds.

To prove Theorem 10, we start with the homogeneous case.

Theorem 11 Let $T$ be a positive constant, $q$ a positive integer, and $c$ a non-negative constant. If $x:[0, T]^{q} \rightarrow \mathbb{R}$ is an integrable function of $q$ variables, satisfying

$$
x\left(t_{1}, \ldots, t_{q}\right) \leqq c \sum_{i=1}^{q} \int_{0}^{t_{i}}\left(\left.x\left(t_{1}, \ldots, t_{q}\right)\right|_{t_{i}=s}\right) d s, \quad\left(t_{1}, \ldots, t_{q}\right) \in[0, T]^{q},
$$

then

$$
x\left(t_{1}, \ldots, t_{q}\right) \leqq 0, \quad\left(t_{1}, \ldots, t_{q}\right) \in[0, T]^{q},
$$

holds.

To prove Theorem [11, we introduce a notation

$$
\begin{aligned}
& \left(A_{i, k} y\right)\left(t_{1}, \ldots, t_{q}\right)
\end{aligned}
$$

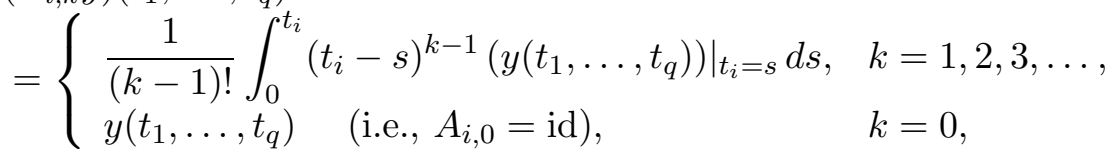

for integrable function $y:[0, T]^{q} \rightarrow \mathbb{R}$ in $q$ variables and $i=1, \ldots, q . A_{i, k}, k \in \mathbb{Z}_{+}, i=1,2, \ldots, q$, are commutative operators on the set of integrable functions. In fact, commutativity is obvious for $k=0$, and by induction in $k$ we have

$$
A_{i, k} A_{i, \ell}=A_{i, 1}^{k+\ell}=A_{i, k+\ell}=A_{i, \ell} A_{i, k}
$$

and Fubini's theorem implies for $k \ell>0$ and $i \neq j$

$$
\begin{aligned}
& \begin{array}{l}
\left(A_{i, k} A_{j, \ell} y\right)\left(t_{1}, \ldots, t_{q}\right) \\
=\frac{1}{(k-1) !} \frac{1}{(\ell-1) !} \int_{0}^{t_{i}} d s \int_{0}^{t_{j}} d u\left(t_{i}-s\right)^{k-1} \\
\quad \times\left.\left(\left.\left(t_{j}-u\right)^{\ell-1}\left(y\left(t_{1}, \ldots, t_{q}\right)\right)\right|_{t_{j}=u}\right)\right|_{t_{i}=s} \\
=\left(A_{j, \ell} A_{i, k} y\right)\left(t_{1}, \ldots, t_{q}\right),
\end{array}
\end{aligned}
$$

which prove

$$
A_{i, k} A_{j, \ell}=A_{j, \ell} A_{i, k}, \quad k, \ell \in \mathbb{Z}_{+}, i, j \in\{1, \ldots, q\}
$$


Lemma 12 Under the assumptions of Theorem 11,

$$
x\left(t_{1}, \ldots, t_{q}\right) \leqq c^{N} \sum_{\substack{\left(k_{1}, \ldots, k_{q}\right) \in \mathbb{Z}_{+}^{q} ; \\ k_{1}+\cdots+k_{q}=N}}\left(A_{q, k_{q}} A_{q-1, k_{q-1}} \cdots A_{1, k_{1}} x\right)\left(t_{1}, \ldots, t_{q}\right), N \in \mathbb{Z}_{+},
$$

holds.

Proof. The case $N=1$ of (91) is the assumption (86) itself. Assume that (91) holds for some $N$. Substituting (86) in (91), and noting that sums, integrations, and multiplication of non-negative reals have monotonicity, we have

$$
x\left(t_{1}, \ldots, t_{q}\right) \leqq c^{N+1} \sum_{i=1}^{q} \sum_{\substack{\left(k_{1}, \ldots, k_{q}\right) \in \mathbb{Z}_{+}^{q} ; \\ k_{1}+\cdots+k_{q}=N}}\left(A_{q, k_{q}} A_{q-1, k_{q-1}} \cdots A_{1, k_{1}} A_{i, 1} x\right)\left(t_{1}, \ldots, t_{q}\right) .
$$

Using (89) in the form $A_{i, k_{i}} A_{i, 1}=A_{i, k_{i}+1}$, we have (87) for $N$ replaced by $N+1$.

Proof of Theorem 11, For notational simplicity, put $\vec{t}=\left(t_{1}, \ldots, t_{q}\right)$ in this proof. The operator $A_{i, k}$ in (88) satisfies

$$
\left(A_{i, k} y\right)(\vec{t}) \leqq \frac{t_{i}^{k}}{k !} \sup _{\vec{t} \in[0, T]^{q}} y(\vec{t}), \quad \vec{t} \in[0, T]^{q}
$$

for a integrable function $y$, hence (91) implies

$$
x(\vec{t}) \leqq c^{N} \sum_{\substack{\left(k_{1}, \ldots, k_{q}\right) \in \mathbb{Z}_{+}^{q} ; \\ k_{1}+\cdots+k_{q}=N}} \prod_{i=1}^{q} \frac{t_{i}^{k_{i}}}{k_{i} !} \sup _{\vec{t} \in[0, T]^{q}} x(\vec{t}), \quad \vec{t} \in[0, T]^{q}, N \in \mathbb{N} .
$$

For an arbitrary $\epsilon>0$, let $\vec{t}_{0}=\left(t_{0,1}, \ldots, t_{0, q}\right) \in[0, T]^{q}$ be a vector (independent of $N$ ) such that $x\left(\vec{t}_{0}\right) \geqq \sup _{\vec{t} \in[0, T]^{q}} x(\vec{t})-\epsilon$ holds. Put

$$
a_{N}=c^{N} \sum_{\substack{\left(k_{1}, \ldots, k_{q}\right) \in \mathbb{Z}_{+}^{q} ; \\ k_{1}+\cdots+k_{q}=N}} \prod_{i=1}^{q} \frac{t_{0, i}^{k_{i}}}{k_{i} !}
$$

Then (92) implies

$$
\sup _{\vec{t} \in[0, T]^{q}} x(\vec{t}) \leqq x\left(\vec{t}_{0}\right)+\epsilon \leqq a_{N} \sup _{\vec{t} \in[0, T]^{q}} x(\vec{t})+\epsilon
$$

hence

$$
\sup _{\vec{t} \in[0, T]^{q}} x(\vec{t})\left(1-a_{N}\right) \leqq \epsilon
$$

holds. We see

$$
\sum_{N=0}^{\infty} a_{N} \leqq \sum_{N=0}^{\infty} \sum_{\substack{\left(k_{1}, \ldots, k_{q}\right) \in \mathbb{Z}_{+}^{q} ; \\ k_{1}+\cdots+k_{q}=N}} \prod_{i=1}^{q} \frac{\left(c t_{0, i}\right)^{k_{i}}}{k_{i} !}=\prod_{i=1}^{q} e^{c t_{0, i}}<\infty
$$


so that, in particular, $\lim _{N \rightarrow \infty} a_{N}=0$, which implies $1-a_{N} \leqq \frac{1}{2}$ for large $N$. Hence

$$
\sup _{\vec{t} \in[0, T]^{q}} x(\vec{t}) \leqq 2 \epsilon
$$

which proves (87).

Proof of Theorem 10. Note that

$$
x_{1}\left(s_{1}, \ldots, s_{q}\right)=a e^{c\left(s_{1}+\cdots+s_{q}\right)}
$$

satisfies

$$
\begin{aligned}
& x_{1}\left(s_{1}, \ldots, s_{q}\right)=e^{-c s_{i}} x_{1}\left(s_{1}, \ldots, s_{q}\right)+c \int_{0}^{s_{i}}\left(\left.x_{1}\left(s_{1}, \ldots, s_{q}\right)\right|_{s_{i}=u}\right) d u, \\
& \left(s_{1}, \ldots, s_{q}\right) \in[0, t]^{q}, i=1,2, \ldots, q .
\end{aligned}
$$

Subtracting $x_{1}\left(s_{1}, \ldots, s_{q}\right)$ from (85), and then using (94), we have

$$
\begin{aligned}
& x\left(t_{1}, \ldots, t_{q}\right)-x_{1}\left(t_{1}, \ldots, t_{q}\right) \leqq\left.\frac{c}{q} \sum_{i=1}^{q} \int_{0}^{t_{i}}\left(x\left(t_{1}, \ldots, t_{q}\right)-x_{1}\left(t_{1}, \ldots, t_{q}\right)\right)\right|_{t_{i}=s} d s, \\
& \left(t_{1}, \ldots, t_{q}\right) \in[0, T]^{q}
\end{aligned}
$$

which, with Theorem 11 and (93) implies (85).

Finally, we give a result to be used in the proof of the main theorem in $\S 5$ which contains recursion with respect to the number of variables $q$ and a nonlinear term.

Theorem 13 Let $T$ be a positive constant, $d$ be a non-negative constant satisfying $d \leqq 1$, and for each positive integer $q$ let $a_{q}, b_{q}$, and $c_{q}$ be non-negative constants. Assume that, for a series of non-negative valued integrable functions $x_{q}:[0, T]^{q} \rightarrow[0, \infty), q \in \mathbb{Z}_{+}$,

$$
\begin{aligned}
& x_{0}=1, \\
& \begin{aligned}
& x_{q}\left(t_{1}, \ldots, t_{q}\right) \leqq a_{q} \sum_{i=1}^{q} x_{q-1}\left(t_{1}, \ldots, t_{h}, \ldots, t_{q}\right)^{d} \\
&+b_{q} \sum_{i=1}^{q} x_{q-1}\left(t_{1}, \ldots, t_{h}, \ldots, t_{q}\right) \\
&+c_{q} \sum_{i=1}^{q} \int_{0}^{t_{i}}\left(\left.x_{q}\left(t_{1}, \ldots, t_{q}\right)\right|_{t_{i}=s}\right) d s, \\
&\left(t_{1}, \ldots, t_{q}\right) \in[0, T]^{q}, \quad q \in \mathbb{N},
\end{aligned}
\end{aligned}
$$

hold. Put

$$
\tilde{c}_{q}=\max _{1 \leqq k \leqq q} k c_{k}, q \in \mathbb{N},
$$

and define a sequence of non-negative constants $g_{q}, q=0,1,2, \ldots$, recursively by

$$
g_{0}=1, \quad g_{q}=q\left(a_{q} g_{q-1}^{d}+b_{q} g_{q-1}\right), q \in \mathbb{N} .
$$

Then

$$
x_{q}\left(t_{1}, \ldots, t_{q}\right) \leqq g_{q} e^{\tilde{c}_{q}\left(t_{1}+\cdots+t_{q}\right)}, \quad\left(t_{1}, \ldots, t_{q}\right) \in[0, T]^{q}, \quad q \in \mathbb{N},
$$

holds. 
Proof. If $q=1$, (95) reads $x_{1}\left(t_{1}\right) \leqq\left(a_{1}+b_{1}\right)+c_{1} \int_{0}^{t_{1}} x_{1}(s) d s$, hence Theorem 9 implies $x_{1}\left(t_{1}\right) \leqq$ $g_{1} e^{c_{1} t_{1}}$, which proves (96) for $q=1$.

Let $q \geqq 2$ and assume that (96) holds for $x_{q-1}$, as

$$
x_{q-1}\left(t_{1}, \ldots, t_{q-1}\right) \leqq g_{q-1} e^{\tilde{c}_{q-1}\left(t_{1}+\cdots+t_{q-1}\right)} .
$$

This and (95) for $x_{q}$ and $d \leqq 1$ imply

$$
\begin{aligned}
x_{q}\left(s_{1}, \ldots, s_{q}\right) \leqq & q\left(a_{q} g_{q-1}^{d}+b_{q} g_{q-1}\right) e^{\tilde{c}_{q-1}\left(s_{1}+\cdots+s_{q}\right)} \frac{1}{q} \sum_{i=1}^{q} e^{-\tilde{c}_{q-1} s_{i}} \\
& +q c_{q} \frac{1}{q} \sum_{i=1}^{q} \int_{0}^{s_{i}}\left(\left.x_{q}\left(s_{1}, \ldots, s_{q}\right)\right|_{s_{i}=u}\right) d u
\end{aligned}
$$

which, with Theorem 10, implies (96) for $x_{q}$.

\section{Proof of the main theorem.}

\subsection{Convergence of the spatial distribution function.}

Here we will prove the essential part of the infinite particle limit, the convergence of spatial distribution function.

In analogy to (36), define, for each $i=1,2, \ldots, N$ and $0 \leqq t_{0} \leqq t \leqq T$ and $0 \leqq y_{0} \leqq 1$,

$$
J_{i}^{(N)}\left(t_{0}, t\right)=\left\{\omega \in \Omega \mid \tilde{\nu}_{i}^{(N)}(t)(\omega)>\tilde{\nu}_{i}^{(N)}\left(t_{0}\right)(\omega)\right\}
$$

By similar arguments as for (48) and (38), $\varphi^{(N)}$ in (86) and $Y_{C}^{(N)}$ in (9) respectively satisfies

$$
\varphi^{(N)}\left(d w,\left(y_{0}, t_{0}\right), t\right)=\frac{1}{N} \sum_{j ; Y_{j}^{(N)}\left(t_{0}\right) \geqq y_{0}} \mathbf{1}_{J_{j}^{(N)}\left(t_{0}, t\right)^{c}} \delta_{w_{j}}(d w),
$$

and

$$
Y_{C}^{(N)}\left(\left(y_{0}, t_{0}\right), t\right)=y_{0}+\frac{1}{N} \sum_{j ; Y_{j}^{(N)}\left(t_{0}\right) \geqq y_{0}} \mathbf{1}_{J_{j}^{(N)}\left(t_{0}, t\right)} .
$$

Theorem 14 Assume (2), (21), (25), and (27). Then there exists $\delta^{\prime}>0$ and an integer $p_{0}$ satisfying $2 p_{0} \delta^{\prime}>1$, such that for any integer $p \geqq p_{0}$ there exists a positive constant $C$ depending only on $p$ and $\delta^{\prime}$, (independent of $N$ and $h$,) such that for any bounded continuous $h: W \rightarrow \mathbb{R}$

$$
\mathrm{E}\left[\sup _{(\gamma, t) \in \Delta_{T}}\left|\varphi^{(N)}(h, \gamma, t)-\varphi_{y_{C}}(h, \gamma, t)\right|^{2 p}\right] \leqq \frac{C C_{h}^{2 p}}{N^{2 p \delta^{\prime}}}, \quad N \in \mathbb{N},
$$

holds, where $C_{h}$ is as in (51). 
Note that (9) and (18) with $\theta=y_{C}$ imply

$$
Y_{C}^{(N)}(\gamma, t)-y_{C}(\gamma, t)=\frac{\left[N\left(1-y_{0}\right)\right]}{N}-\left(1-y_{0}\right)+\varphi_{y_{C}}(W, \gamma, t)-\varphi^{(N)}(W, \gamma, t) .
$$

Applying $(a+b)^{2 p} \leqq 2^{2 p-1}\left(a^{2 p}+b^{2 p}\right)$, valid for $a, b \geqq 0$ and $2 p \geqq 1$, Theorem 14 with $h(w)=1$, $w \in W$, therefore implies

$$
\mathrm{E}\left[\sup _{(\gamma, t) \in \Delta_{T}}\left|Y_{C}^{(N)}(\gamma, t)-y_{C}(\gamma, t)\right|^{2 p}\right] \leqq \frac{2^{2 p-1} C}{N^{2 p \delta^{\prime}}}+\frac{2^{2 p-1}}{N^{2 p}}, N \in \mathbb{N},
$$

with the assumptions and notations of the Theorem.

\subsubsection{Coupling of the original and the flow driven model.}

In view of Theorem 4, it suffices to prove the following for Theorem 14 to hold.

Theorem 15 Assume (2), (21), (25), and (27), and let $\delta$ be as in (21). Then there exists $\delta^{\prime}>0$ and an integer $p_{0}$ satisfying $2 p_{0} \delta^{\prime}>1$, such that for any integer $p \geqq p_{0}$ there exists a positive constant $C$, (independent of $N$ and $h$,) such that for any bounded continuous $h: W \rightarrow \mathbb{R}$

$$
\mathrm{E}\left[\sup _{\left(\left(y_{0}, t_{0}\right), t\right) \in \Delta_{T}}\left|\varphi^{(N)}(h, \gamma, t)-\varphi^{\left(N, y_{C}\right)}(h, \gamma, t)\right|^{2 p}\right] \leqq \frac{C C_{h}^{2 p}}{N^{2 p \delta^{\prime}}}, \quad N \in \mathbb{N},
$$

where $C_{h}$ is as in (51).

For $t \in[0, T]$ and $i \in\{1,2, \ldots, N\}$, put

$$
\tilde{w}_{i, \wedge}^{(N)}(t)=w_{i}\left(Y_{i}^{(N)}(t-), t\right) \wedge w_{i}\left(y_{C}\left(\gamma_{i}^{\left(N, y_{C}\right)}(t-), t\right), t\right),
$$

$$
\tilde{w}_{i, \vee}^{(N)}(t)=w_{i}\left(Y_{i}^{(N)}(t-), t\right) \vee w_{i}\left(y_{C}\left(\gamma_{i}^{\left(N, y_{C}\right)}(t-), t\right), t\right),
$$

and denote the event that the $i$-th particle $Y_{i}^{(N)}(s)$ of (3) and $Y_{i}^{\left(N, y_{C}\right)}(s)$ of (35) jump to top at same times in the interval $\left(t_{0}, t\right]$ by

$$
\mathcal{K}_{i}^{(N)}\left(t_{0}, t\right)=\left\{\omega \in \Omega \mid \nu_{i}^{(N)}\left(\left\{(s, \xi) \mid \tilde{w}_{i, \wedge}^{(N)}(s)<\xi \leqq \tilde{w}_{i, \vee}^{(N)}(s), s \in\left(t_{0}, t\right]\right\}\right)=0\right\} .
$$

Fix a bounded continuous function $h: W \rightarrow \mathbb{R}$, and let $C_{h}$ be as in (51). Using the definitions (98) and (53), put

$$
\begin{aligned}
\Delta \varphi^{(N)}(\gamma, t) & =\varphi^{(N)}(h, \gamma, t)-\varphi^{(N, \theta)}(h, \gamma, t) \\
& =\frac{1}{N} \sum_{j ; y_{j}^{(N)} \geqq y_{0}} h\left(w_{j}\right)\left(\mathbf{1}_{J_{j}^{(N)}\left(t_{0}, t\right)^{c}}-\mathbf{1}_{J_{j}^{(N, \theta)}\left(t_{0}, t\right)^{c}}\right), \\
\gamma=\left(y_{0}, t_{0}\right) & \in \Gamma_{t}, t \in[0, T] .
\end{aligned}
$$

Then (105) and (51) imply,

$$
\left|\Delta \varphi^{(N)}(\gamma, t)\right| \leqq \frac{C_{h}}{N} \sum_{j=1}^{N} \mathbf{1}_{\mathcal{K}_{j}^{(N)}\left(t_{0}, t\right)^{c}}, \quad \gamma=\left(y_{0}, t_{0}\right) \in \Gamma_{t}, t \in[0, T] .
$$

The monotonicity of $\mathcal{K}_{j}^{(N)}\left(t_{0}, t\right)^{c}$ with respect to $t$ and $t_{0}$ further implies

$$
\sup _{(\gamma, t) \in \Delta_{T}}\left|\Delta \varphi^{(N)}(\gamma, t)\right| \leqq \frac{C_{h}}{N} \sum_{i=1}^{N} \mathbf{1}_{\mathcal{K}_{i}^{(N)}(0, T)^{c}},
$$

Proof of Theorem 15 therefore reduces to evaluation of the event $\mathcal{K}_{i}^{(N)}(0, T)$. 


\subsubsection{Event with different jumps to top.}

As an analog of (132), define a sequence of stopping times, $0=\tau_{i, 0}^{(N)}<\tau_{i, 1}^{(N)}<\cdots$, by

$$
\begin{aligned}
& \tau_{i, 0}^{(N)}=0 \\
& \tau_{i, k+1}^{(N)}=\inf \left\{t>\tau_{i, k}^{(N)} \mid \nu_{i}^{(N)}\left(\left\{(s, \xi) \in\left(\tau_{i, k}^{(N)}, T\right] \times[0, \infty) \mid\right.\right.\right. \\
& \left.\left.\left.0 \leqq \xi \leqq w_{i}\left(Y_{i}^{(N)}(s-), s\right)\right\}\right)>0\right\}, k \in \mathbb{Z}_{+} .
\end{aligned}
$$

$\tau_{i, k}^{(N)}$ is the time that the particle $i$ in the (original) stochastic ranking process jumps to the top for the $k$-th time. A corresponding analog of (33) is

$$
\gamma_{i}^{(N)}(t)= \begin{cases}\left(y_{i}^{(N)}, 0\right), & 0 \leqq t<\tau_{i, 1}^{(N)} \\ \left(0, \tau_{i, k}^{(N)}\right), & \tau_{i, k}^{(N)} \leqq t<\tau_{i, k+1}^{(N)}, k=1,2, \ldots\end{cases}
$$

A property corresponding to (42) then is

$$
Y_{i}^{(N)}(t)=Y_{C}^{(N)}\left(\gamma_{i}^{(N)}(t), t\right), t \in[0, T],
$$

which can be proved in a similar way as a proof of (42) in Lemma 3. This decomposition in particular decomposes the dependence as random variables; if we temporarily denote by $X \in \mathcal{F}$, a fact that a random variable $X: \Omega \rightarrow \mathbb{R}$ is $\mathcal{F}$-measurable, and denote by $\sigma[Z]$ a sigma algebra generated by a random variable $Z$, we have

$$
\begin{aligned}
& Y_{C}^{(N)}\left(\left(y_{0}, t_{0}\right), t\right) \in \sigma\left[\left\{\nu_{j}^{(N)} \mid Y_{j}^{(N)}\left(t_{0}\right)>y_{0}\right\}\right], \\
& \gamma_{i}^{(N)} \in \sigma\left[\left\{\tau_{i, k}^{(N)} \wedge t \mid k \in \mathbb{N}\right\}\right] .
\end{aligned}
$$

Define an analog of the stopping times (108) using (103) by

$$
\begin{aligned}
& \tau_{i, \wedge, 0}^{(N)}=0 \\
& \tau_{i, \wedge, k}^{(N)}=\inf \left\{t>\tau_{i, \wedge, k-1}^{(N)} \mid \nu_{i}^{(N)}\left(\left\{(\xi, s) \mid 0 \leqq \xi \leqq \tilde{w}_{i, \wedge}^{(N)}(s), 0 \leqq s \leqq t\right\}\right)>0\right\}, \\
& k \in \mathbb{N}
\end{aligned}
$$

and denote by $\sigma_{i}^{(N)}$, the time that the particle pair with label $i$ of the original model and the flow driven model have different jumps to the top for the first time;

$$
\sigma_{i}^{(N)}=\inf \left\{t \in[0, T] \mid \mathcal{K}_{i}^{(N)}(0, t)^{c}\right\}
$$

The definition implies

$$
\tau_{i, k}^{(N)}<\sigma_{i}^{(N)} \Rightarrow \tau_{i, \wedge, k}^{(N)}=\tau_{i, k}^{(N)}=\tau_{i, k}^{\left(N, y_{C}\right)},
$$

where $\tau_{i, k}^{\left(N, y_{C}\right)}$ is defined in (32), with $\theta=y_{C}$. Indepndence of $\nu_{i}^{(N)}(A)$ and $\nu_{i}^{(N)}(B)$ for the exclusive events $A$ and $B$ implies

$$
\left\{\tau_{i, \wedge, k}^{(N)} \mid k \in \mathbb{Z}_{+}\right\} \perp \sigma_{i}^{(N)} .
$$

Using (104), (103), (2) $\mathrm{C}$ and $a \vee b=|a-b|+a \wedge b$, we have

$$
\begin{gathered}
\left\{(\xi, s) \in \mathbb{R}^{2} \mid \tilde{w}_{i, \wedge}^{(N)}(s)<\xi \leqq \tilde{w}_{i, \vee}^{(N)}(s), 0<s \leqq t\right\} \\
\subset\left\{(\xi, s) \in \mathbb{R}^{2}\left|0 \leqq \xi-\tilde{w}_{i, \wedge}^{(N)}(s) \leqq C_{W}\right| Y_{i}^{(N)}(s)-y_{C}\left(\gamma_{i}^{\left(N, y_{C}\right)}(s), s\right) \mid\right. \\
0<s \leqq t\} \\
\subset \bigcup_{k=1}^{\infty}\left\{(\xi, s) \in \mathbb{R}^{2}\left|0 \leqq \xi-\tilde{w}_{i, \wedge}^{(N)}(s) \leqq C_{W}\right| Y_{i}^{(N)}(s)-y_{C}\left(\gamma_{i}^{\left(N, y_{C}\right)}(s), s\right) \mid,\right. \\
\left.t \wedge \tau_{i, \wedge, k-1}^{(N)}<s \leqq t \wedge \tau_{i, \wedge, k}^{(N)}\right\} .
\end{gathered}
$$


Note that for each $i$

$$
Y_{i}^{(N)}\left(\tau_{i, k-1}^{(N)}\right)=Y_{i}^{\left(N, y_{C}\right)}\left(\tau_{i, k-1}^{(N)}\right)=0, \quad \text { on } \tau_{i, k-1}^{(N)}<\sigma_{i}^{(N)}
$$

Note also that the definition (113) implies $\left\{\sigma_{i}^{(N)}>s\right\}=\mathcal{K}_{i}^{(N)}(0, s)$. Hence, (110), (42), (199), and (38) imply, with similar arguments for deriving (107) from (105),

$$
\begin{aligned}
\left|Y_{i}^{(N)}(s)-Y_{i}^{\left(N, y_{C}\right)}(s)\right| & =\left|Y_{C}^{(N)}\left(\gamma_{i}^{(N)}(s), s\right)-Y_{C}^{\left(N, y_{C}\right)}\left(\gamma_{i}^{(N)}(s), s\right)\right| \\
& =\frac{1}{N}\left|\sum_{j \neq i}\left(\mathbf{1}_{\left(J_{j}^{(N)}\left(\tau_{i, k-1}^{(N)}, s\right)\right)^{c}}-\mathbf{1}_{\left(J_{j}^{(N)}\left(\tau_{i, k-1}^{\left(N, y_{C}\right)}, s\right)\right)^{c}}\right)\right| \\
& \leqq \frac{1}{N} \sum_{j \neq i} \mathbf{1}_{\mathcal{K}_{j}^{(N)}\left(\tau_{i, k-1}^{(N)}, s\right)^{c}} \\
\text { on } \mathcal{K}_{i}^{(N)}(0, s), \tau_{i, k-1}^{(N)} & \leqq s<\tau_{i, k}^{(N)} .
\end{aligned}
$$

This with (116) then implies

$$
\begin{aligned}
\mathcal{K}_{i}^{(N)}(0, t)^{c} & \\
\subset \bigcup_{k=1}^{\infty}\left(\left\{\omega \in \Omega \mid \nu_{i}^{(N)}(\{(s, \xi) \mid 0 \leqq\right.\right. & -\tilde{w}_{i, \wedge}^{(N)}(s) \\
\leqq & C_{W}\left|Y_{i}^{\left(N, y_{C}\right)}(s)-y_{C}\left(\gamma_{i}^{\left(N, y_{C}\right)}(s), s\right)\right| \\
& +\frac{C_{W}}{N} \sum_{j \neq i} \mathbf{1}_{\mathcal{K}_{j}^{(N)}\left(\tau_{i, k-1}^{(N)}, s\right)^{c}}, \\
& \left.\left.\left.t \wedge \tau_{i, \wedge, k-1}^{(N)}<s \leqq t \wedge \tau_{i, \wedge, k}^{(N)}\right\}\right)>0\right\} \\
\left.\cap \mathcal{K}_{i}^{(N)}\left(0, \tau_{i, k-1}^{(N)}\right)\right) . &
\end{aligned}
$$

\subsubsection{Application of Gronwall hierarchy.}

For $q=1,2, \ldots, N$ and $t_{i} \in[0, T], i=1, \ldots, q$, put

$$
X_{q}^{(N)}\left(t_{1}, \ldots, t_{q}\right)=\max _{\left\{i_{1}, \ldots, i_{q}\right\} \subset\{1, \ldots, N\}} \mathrm{E}\left[\prod_{\alpha=1}^{q} \mathbf{1}_{\mathcal{K}_{i_{\alpha}}^{(N)}\left(0, t_{\alpha}\right)^{c}}\right] .
$$

(106), (107), and (118) imply that to prove Theorem [15, it suffices to find $\delta^{\prime}>0$ and integer $p_{0}$ satisfying $2 p_{0} \delta^{\prime}>1$, such that for any integer $p \geqq p_{0}$,

$$
\frac{1}{N^{2 p}} \sum_{q=1}^{2 p}{ }_{N} \mathrm{C}_{q} d(2 p, q) X_{q}^{(N)}(T, \ldots, T) \leqq \frac{C}{N^{2 p \delta^{\prime}}},
$$

for some $C>0$ independent of $N$. Here, $d(r, q)$ is the number of surjections from a finite set of size $r$ to a set of size $q$, which is determined inductively by

$$
d(r, 1)=1, \text { and } d(r, q)=q^{r}-\sum_{k=1}^{q-1}{ }_{q} \mathrm{C}_{k} d(r, q-k), q=2,3, \ldots, r .
$$


Fix $q$ and $\left\{i_{1}, \ldots, i_{q}\right\}$ in the right hand side of (118). Let $\alpha \in\{1, \ldots, q\}$ be the suffix such that $y_{i_{\alpha}}^{(N)}$ is the smallest among $y_{i_{1}}^{(N)}, \ldots, y_{i_{q}}^{(N)}$, and put $i_{0}=i_{\alpha}$. At times $\tau_{i_{0}, k}^{(N)}, k \in \mathbb{Z}_{+}$, the particle $i_{0}$ is at the top position, namely, for $i_{\alpha} \neq i_{0}$,

$$
\begin{aligned}
& Y_{i_{0}}^{(N)}\left(\tau_{i_{0}, k}^{(N)}\right)=0<Y_{i_{\alpha}}^{(N)}\left(\tau_{i_{0}, k}^{(N)}\right), k=1,2, \ldots, \\
& Y_{i_{0}}^{(N)}\left(\tau_{i_{0}, 0}^{(N)}\right)=Y_{i_{0}}^{(N)}(0)=y_{i_{0}}^{(N)}<y_{i_{\alpha}}^{(N)}=Y_{i_{\alpha}}^{(N)}\left(\tau_{i_{0}, 0}^{(N)}\right) .
\end{aligned}
$$

Hence up to the first jump to the top, each $Y_{i_{\alpha}}^{(N)}(t)$ with $i_{\alpha} \neq i_{0}$ is independent of $\nu_{i_{0}}$. Therefore,

$$
\begin{aligned}
& \mathrm{E}\left[\prod_{\alpha=1}^{q} \mathbf{1}_{\mathcal{K}_{i_{\alpha}}^{(N)}\left(0, t_{\alpha}\right)^{c}}\right] \\
& =\mathrm{E}\left[\prod_{\alpha ; i_{\alpha} \neq i_{0}} \mathbf{1}_{\left(\mathcal{K}_{i_{\alpha}}^{(N)}\left(0, t_{\alpha}\right)\right)^{c}} \mathrm{P}\left[\left(\mathcal{K}_{i_{0}}^{(N)}\left(0, t_{0}\right)\right)^{c} \mid\left\{\nu_{j}, j \neq i_{0}\right\} \cup\left\{\tau_{i_{0}, \wedge, k}^{(N)}\right\}\right]\right],
\end{aligned}
$$

where $\mathrm{P}\left[\cdot \mid\left\{\nu_{j}, j \neq i_{0}\right\} \cup\left\{\tau_{i_{0}, \wedge, k}^{(N)}\right\}\right]$ denotes conditional probability conditioned on the sigma algebra generated by $\nu_{j}, j \neq i_{0}$, and $\tau_{i_{0}, \wedge, k}^{(N)}, k \in \mathbb{N}$. This with (115), (114), (117), and

$$
\mathrm{P}[\nu(A)>0]=1-e^{-|A|} \leqq|A|
$$

for a unit Poisson random measure $\nu$, further leads to

$$
\begin{aligned}
& \mathrm{E}\left[\prod_{\alpha=1}^{q} \mathbf{1}_{\mathcal{K}_{i_{\alpha}}^{(N)}\left(0, t_{\alpha}\right)^{c}}\right] \\
& \leqq \mathrm{E}\left[\prod_{\alpha ; i_{\alpha} \neq i_{0}} \mathbf{1}_{\left(\mathcal{K}_{i_{\alpha}}^{(N)}\left(0, t_{\alpha}\right)\right)^{c}} \sum_{k=1}^{\infty} \mathbf{1}_{\mathcal{K}_{i_{0}}^{(N)}\left(0, t_{0} \wedge \tau_{i_{0}, k-1}^{(N)}\right)}\right. \\
& \times\left(\int_{t_{0} \wedge \tau_{i_{0}, \wedge, k-1}^{(N)}}^{t_{0} \wedge \tau_{i_{0} \wedge, k}^{(N)}} C_{W}\left|Y_{i_{0}}^{\left(N, y_{C}\right)}(s)-y_{C}\left(\gamma_{i_{0}}^{\left(N, y_{C}\right)}(s), s\right)\right| d s\right. \\
& \left.\left.+\int_{t_{0} \wedge \tau_{i_{0}, \wedge, k-1}^{(N)}}^{t_{0} \wedge \tau_{i_{0}, \wedge, k}^{(N)}} \frac{C_{W}}{N} \sum_{j \neq i_{0}} \mathbf{1}_{\mathcal{K}_{j}^{(N)}\left(t_{0} \wedge \tau_{i, k-1}^{(N)}, s\right)^{c}} d s\right)\right] \\
& \leqq \frac{C_{W}}{N} \sum_{j=1}^{N} \mathrm{E}\left[\prod_{\alpha ; i_{\alpha} \neq i_{0}} \mathbf{1}_{\left(\mathcal{K}_{i_{\alpha}}^{(N)}\left(0, t_{\alpha}\right)\right)^{c}} \sum_{k=1}^{\infty} \mathbf{1}_{\mathcal{K}_{i_{0}}^{(N)}\left(0, t_{0} \wedge \tau_{i_{0}, \wedge, k-1}^{(N)}\right)}\right. \\
& \left.\times \int_{t_{0} \wedge \tau_{i_{0}, \wedge, k-1}^{(N)}}^{t_{0} \wedge \tau_{i_{0}, \wedge, k}^{(N)}} \mathbf{1}_{\mathcal{K}_{j}^{(N)}\left(t_{0} \wedge \tau_{i_{0}, k-1}^{(N)}, s-\right)^{c}} d s\right] \\
& +C_{W} \mathrm{E}\left[\prod_{\alpha ; i_{\alpha} \neq i_{0}} \mathbf{1}_{\left(\mathcal{K}_{i_{\alpha}}^{(N)}\left(0, t_{\alpha}\right)\right)^{c}} \sum_{k=1}^{\infty} \mathbf{1}_{\mathcal{K}_{i_{0}}^{(N)}\left(0, t_{0} \wedge \tau_{i_{0}, \wedge, k-1}^{(N)}\right)}\right. \\
& \left.\times \int_{t_{0} \wedge \tau_{i_{0}, \wedge, k-1}^{(N)}}^{t_{0} \wedge \tau_{i_{0}, \wedge, k}^{(N)}}\left|Y_{i_{0}}^{\left(N, y_{C}\right)}(s)-y_{C}\left(\gamma_{i_{0}}^{\left(N, y_{C}\right)}(s), s\right)\right| d s\right] .
\end{aligned}
$$

Using $\mathbf{1}_{\mathcal{K}_{i_{0}}^{(N)}\left(0, t_{0} \wedge \tau_{i_{0}, k-1}^{(N)}\right)} \leqq 1$ and $\mathbf{1}_{\mathcal{K}_{j}^{(N)}\left(t_{0} \wedge \tau_{i_{0}, k-1}^{(N)}, s-\right)^{c}} \leqq \mathbf{1}_{\mathcal{K}_{j}^{(N)}(0, s-)^{c}}$, we see that for $i, j=$ $1,2, \ldots, N$,

$$
\sum_{k \geqq 1} \mathbf{1}_{\mathcal{K}_{i}^{(N)}\left(0, t \wedge \tau_{i, k-1}^{(N)}\right)} \int_{t \wedge \tau_{i, k-1}^{(N)}}^{t \wedge \tau_{i, k}^{(N)}} \mathbf{1}_{\mathcal{K}_{j}^{(N)}\left(t \wedge \tau_{i, k-1}^{(N)}, s-\right)^{c}} d s \leqq \int_{0}^{t} \mathbf{1}_{\mathcal{K}_{j}^{(N)}(0, s-)^{c}} d s
$$


Substituting (122) in the first term of the right hand side of (121), and bounding the characteristic function on the right hand side by 1 for $j \in\left\{i_{1}, \ldots, i_{q}\right\} \backslash\left\{i_{0}\right\}$, and bounding the characteristic function for $i_{0}$ also by 1 in the second term of the right hand side of (121), we have

$$
\begin{aligned}
& X_{q}^{(N)}\left(t_{1}, \ldots, t_{q}\right) \\
& \leqq \frac{C_{W} T(q-1)}{N} \sum_{i_{0}=1}^{q} X_{q-1}^{(N)}\left(t_{1}, \ldots, t \hat{h}_{0}, \ldots, t_{q}\right) \\
& +C_{W} \sum_{i_{0}=1}^{q} \int_{0}^{t_{i_{0}}} X_{q}^{(N)}\left(t_{1}, \ldots, s, \ldots, t_{q}\right) d s \\
& +C_{W} \max _{\left\{i_{1}, \ldots, i_{q}\right\} \subset\{1, \ldots, N\}} \int_{0}^{t_{i_{0}}} \\
& \mathrm{E}\left[\prod_{i_{\alpha} \neq i_{0}}^{\mathbf{1}_{\mathcal{K}_{i_{\alpha}}}\left(0, t_{\alpha}\right)^{c}} \mid Y_{i_{i_{0}}}^{\left(N, y_{C}\right)}(s)-y_{C}\left(\left(\gamma_{i_{i_{0}}}^{\left(N, y_{C}\right)}(s), s\right) \mid\right] d s .\right.
\end{aligned}
$$

Here, $X_{q-1}^{(N)}\left(t_{1}, \ldots, t_{i_{0}}, \ldots, t_{q}\right)$ is the function in (118) with $q$ replaced by $q-1$ and with $q-1$ variables obtained by excluding $t_{i_{0}}$ from $t_{1}, \ldots, t_{q}$, and the variables for $X_{q}^{(N)}\left(t_{1}, \ldots, s, \ldots, t_{q}\right)$ is $t_{1}, \ldots, t_{q}$ with $t_{i_{0}}$ replaced by $s$. Applying Hölder's inequality in the form

$$
\mathrm{E}[|X Y|] \leqq \mathrm{E}\left[|X|^{2 p /(2 p-1)}\right]^{1-(2 p)^{-1}} \mathrm{E}\left[|Y|^{2 p}\right]^{1 /(2 p)}
$$

to the last term in the right hand side of (123), and using (42) and (156), we have

$$
\begin{aligned}
& X_{q}^{(N)}\left(t_{1}, \ldots, t_{q}\right) \\
& \leqq \\
& \quad \frac{C_{W} T(q-1)}{N} \sum_{i_{0}=1}^{q} X_{q-1}^{(N)}\left(t_{1}, \ldots, t \hat{h}_{0}, \ldots, t_{q}\right) \\
& \quad+C_{W} \sum_{i_{0}=1}^{q} \int_{0}^{t_{i_{0}}} X_{q}^{(N)}\left(t_{1}, \ldots, s, \ldots, t_{q}\right) d s \\
& \quad+\frac{C_{W} T C}{N^{\delta}} \sum_{i_{0}=1}^{q}\left(X_{q-1}^{(N)}\left(t_{1}, \ldots, t_{i_{0}}, \ldots, t_{q}\right)\right)^{(2 p-1) /(2 p)}, \\
& q=1,2, \ldots, \\
& X_{0}^{(N)}=1 .
\end{aligned}
$$

Applying Theorem 13, with $a_{q}=C_{W} T C N^{-\delta}, b_{q}=C_{W} T(q-1) N^{-1}, c_{q}=C_{W}, d=1-(2 p)^{-1}$, we have

$$
\begin{aligned}
& X_{q}^{(N)}\left(t_{1}, \ldots, t_{q}\right) \leqq g_{q} e^{q^{2} C_{W} T}, \quad t_{i} \in[0, T], i=1, \ldots, q, q \in \mathbb{N} \\
& g_{0}=1, \quad g_{q}=q C_{W} T\left(C N^{-\delta} g_{q-1}^{d}+(q-1) N^{-1} g_{q-1}\right), q \in \mathbb{N} .
\end{aligned}
$$

For large $N$ we have $g_{q-1}<1$, hence with $d<1$, we further have $g_{q-1}^{d}>g_{q-1}$, and also with $0<\delta<1$ for large $N$ we have $N^{-\delta}>(q-1) N^{-1}$, so that

$$
g_{q} \leqq q C_{W} T g_{q-1}^{d} N^{-\delta}(C+1), q \in \mathbb{N} .
$$

By induction in $q$,

$$
g_{q} \leqq q !\left(C_{W} T \vee 1\right)^{q}(C+1)^{q} \frac{1}{N^{2 p \delta\left(1-\left(1-(2 p)^{-1}\right)^{q}\right)}}, q \in \mathbb{N}
$$


Since $1-\left(1-\frac{1}{2 p}\right) q$ is decreasing in $q$, we therefore have

$$
\begin{aligned}
& X_{q}^{(N)}\left(t_{1}, \ldots, t_{q}\right) \leqq q !\left(C_{W} T \vee 1\right)^{q}(C+1)^{q} e^{q^{2} C_{W} T} \frac{1}{N^{2 p \delta\left(1-\left(1-(2 p)^{-1}\right)^{2 p}\right)}}, \\
& q=1,2, \ldots, p .
\end{aligned}
$$

Choose $\delta^{\prime}$ to be any positive constant satisfying

$$
0<\delta^{\prime}<\left(1-\frac{1}{e}\right) \delta
$$

Since $\lim _{p \rightarrow \infty}\left(1-\frac{1}{2 p}\right)^{2 p}=e^{-1}<1$, there exists an integer $p_{0}>\frac{1}{2 \delta^{\prime}}$ such that

$$
\delta^{\prime}<\left(1-\left(1-\frac{1}{2 p}\right)^{2 p}\right) \delta, \quad p=p_{0}, p_{0}+1, \ldots
$$

With (125) we arrive at

$$
X_{q}^{(N)}\left(t_{1}, \ldots, t_{q}\right) \leqq q !\left(C_{W} T \vee 1\right)^{q}(C+1)^{q} e^{q^{2} C_{W} T} \frac{1}{N^{2 p \delta^{\prime}}}, q=1,2, \ldots, 2 p,
$$

for $p=p_{0}, p_{0}+1, \ldots$. Since ${ }_{N} \mathrm{C}_{q} \leqq \frac{N^{q}}{q !} \leqq \frac{N^{2 p}}{q !}$, this proves (119).

This completes a proof of Theorem [15, and therefore, of Theorem 14.

\subsection{Proof of Theorem 2 ,}

Let $y \in[0,1]$ and let $h: W \rightarrow \mathbb{R}$ be a bounded continuous function with $C_{h}>0$ as in (51). For the flow $y_{C} \in \Theta_{T}$ in Theorem 1 the definition of $\Theta_{T}$ in (13) implies that for each $t \in[0, T]$, $\Gamma_{t} \ni \gamma \mapsto y_{C}(\gamma, t) \in[0,1]$ is surjective. Therefore there exists $\gamma_{t}:[0,1] \rightarrow \Gamma_{t}$ such that

$$
y_{C}\left(\gamma_{t}(y), t\right)=y, y \in[0,1] \text {. }
$$

We then have, using (19), (154), (126), (8), (18), (151), (15), and (126) in turn,

$$
\begin{aligned}
&\left|\int_{W} h(w) \mu_{t}^{(N)}(d w \times[y, 1])-\int_{W} h(w) \mu_{t}(d w \times[y, 1])\right| \\
&=\left|\int_{W} h(w) \mu_{t}^{(N)}(d w \times[y, 1])-\varphi_{y_{C}}\left(h, \gamma_{t}(y), t\right)\right| \\
& \leqq \sup _{\gamma \in \Gamma_{t}}\left|\varphi^{(N)}(h, \gamma, t)-\varphi_{y_{C}}(h, \gamma, t)\right| \\
&+\left|\int_{W} h(w) \mu_{t}^{(N)}(d w \times[y, 1])-\int_{W} h(w) \mu_{t}^{(N)}\left(d w \times\left[Y_{C}^{(N)}\left(\gamma_{t}(y), t\right), 1\right]\right)\right| \\
& \leqq \sup _{\gamma \in \Gamma_{t}}\left|\varphi^{(N)}(h, \gamma, t)-\varphi_{y_{C}}(h, \gamma, t)\right|+C_{h}\left|y-Y_{C}^{(N)}\left(\gamma_{t}(y), t\right)\right| \\
& \leqq \sup _{\gamma \in \Gamma_{t}}\left|\varphi^{(N)}(h, \gamma, t)-\varphi_{y_{C}}(h, \gamma, t)\right|+C_{h}\left|y_{C}\left(\gamma_{t}(y), t\right)-Y_{C}^{(N)}\left(\gamma_{t}(y), t\right)\right| \\
& \leqq \sup _{\gamma \in \Gamma_{t}}\left|\varphi^{(N)}(h, \gamma, t)-\varphi_{y_{C}}(h, \gamma, t)\right|+C_{h} \sup _{\gamma \in \Gamma_{t}}\left|y_{C}(\gamma, t)-Y_{C}^{(N)}(\gamma, t)\right| .
\end{aligned}
$$

Theorem 14 and (101) then imply

$$
\begin{aligned}
& \mathrm{E}\left[\sup _{t \in[0, T]}\left|\int_{W} h(w) \mu_{t}^{(N)}(d w \times[y, 1])-\int_{W} h(w) \mu_{t}(d w \times[y, 1])\right|^{2 p}\right] \\
& \leqq \frac{\left(1+2^{2 p-1} C_{h}\right) C}{N^{2 p \delta^{\prime}}}+\frac{2^{2 p-1} C_{h}}{N^{2 p}}, \quad N \in \mathbb{N}
\end{aligned}
$$


where the constants in the right hand side is as in Theorem [14. Since $2 p \delta^{\prime}>1$, we see that

$$
\mathrm{E}\left[\sum_{N=1}^{\infty} \sup _{t \in[0, T]}\left|\int_{W} h(w) \mu_{t}^{(N)}(d w \times[y, 1])-\int_{W} h(w) \mu_{t}(d w \times[y, 1])\right|^{2 p}\right]<\infty
$$

hence, in particular, we have

$$
\lim _{N \rightarrow \infty} \sup _{t \in[0, T]}\left|\int_{W} h(w) \mu_{t}^{(N)}(d w \times[y, 1])-\int_{W} h(w) \mu_{t}(d w \times[y, 1])\right|=0,
$$

with probability 1 , which proves (28).

Next we prove uniform almost sure convergence of $Y_{i}^{(N)}$ to $Y_{i}$ for $i=1,2, \ldots, L$. As an analogy to (32) and (33), define

$$
\begin{aligned}
& \tau_{i, 0}=0 \\
& \tau_{i, k+1}=\inf \left\{t>\tau_{i, k} \mid \nu_{i}\left(\left\{(s, \xi) \in\left(\tau_{i, k}, T\right] \times[0, \infty) \mid\right.\right.\right. \\
& \left.\left.\left.\quad 0 \leqq \xi \leqq w_{i}\left(Y_{i}(s-), s\right)\right\}\right)>0\right\}, k \in \mathbb{Z}_{+},
\end{aligned}
$$

and

$$
\gamma_{i}(t)= \begin{cases}\left(y_{i}, 0\right), & 0 \leqq t<\tau_{i, 1}, \\ \left(0, \tau_{i, k}\right), & \tau_{i, k} \leqq t<\tau_{i, k+1}, k=1,2, \ldots\end{cases}
$$

Comparing (20) and (30), we have, with similar argument for (42),

$$
Y_{i}(t)=y_{C}\left(\gamma_{i}(t), t\right), t \in[0, T]
$$

Quantities corresponding to (103), (104), (105), and (113) are

$$
\begin{aligned}
& \left.\breve{w}_{i, \wedge}^{(N)}(t)=w_{i}\left(Y_{i}^{(N)}(t-), t\right) \wedge w_{i}\left(Y_{i}(t-), t\right), t\right) \\
& \left.\breve{w}_{i, \mathrm{~N}}^{(N)}(t)=w_{i}\left(Y_{i}^{(N)}(t-), t\right) \vee w_{i}\left(Y_{i}(t-), t\right), t\right), \\
& \tilde{\mathcal{K}}_{i}^{(N)}(0, t)=\left\{\omega \in \Omega \mid \nu_{i}\left(\left\{(s, \xi) \mid \breve{w}_{i, \wedge}^{(N)}(s)<\xi \leqq \breve{w}_{i, \vee}^{(N)}(s), s \in(0, t]\right\}\right)=0\right\}, \\
& \tilde{\sigma}_{i}^{(N)}=\inf \left\{t \in[0, T] \mid \tilde{\mathcal{K}}_{i}^{(N)}(0, t)^{c}\right\} .
\end{aligned}
$$

A proof now proceeds with argument similar to that in $\S 5.1 .2$. An argument similar to that for (116) leads to a bound

$$
\begin{aligned}
& \tilde{\mathcal{K}}_{i}^{(N)}(0, t)^{c} \\
& \subset\left\{\omega \in \Omega \mid \nu_{i}\left(\left\{(s, \xi)\left|0 \leqq \xi-\breve{w}_{i, \wedge}^{(N)}(s) \leqq C_{W}\right| Y_{i}^{(N)}(s)-Y_{i}(s) \mid,\right.\right.\right. \\
& \quad s \in(0, t]\})>0\} .
\end{aligned}
$$

Since $\nu_{i}$ is a Poisson random measure, it holds with probability 1 that $\nu_{i}(A)<\infty$ for a Borel set $A \subset[0, T] \times[0, \infty)$ of finite area. Hence for almost all $\omega \in \Omega$ there exists $\epsilon=\epsilon(\omega)>0$ such that

$$
\nu_{i}\left(\left\{(s, \xi) \mid 0 \leqq \xi-\breve{w}_{i, \wedge}^{(N)}(s) \leqq \epsilon, s \in(0, t]\right\}\right)=0
$$

On the other hand, applying the argument from (127) to (128), (101) implies

$$
\lim _{N \rightarrow \infty} \sup _{(\gamma, t) \in \Delta_{T}}\left|Y_{C}^{(N)}(\gamma, t)-y_{C}(\gamma, t)\right|=0, \text { a.s.. }
$$


Therefore, for almost all $\omega \in \Omega$, there exists an integer $N_{0}=N_{0}(\omega)$ such that for $N \geqq N_{0}$,

$$
\sup _{(\gamma, t) \in \Delta_{T}}\left|Y_{C}^{(N)}(\gamma, t)-y_{C}(\gamma, t)\right| \leqq \frac{\epsilon}{C_{W}}, N \geqq N_{0} .
$$

Combining (133), (135), (110), (131), and (132),

$$
\tilde{\mathcal{K}}_{i}^{(N)}(0, T)^{c}=\emptyset, N \geqq N_{0} .
$$

Next, (110) and (131) imply

$$
\begin{aligned}
& \left|Y_{i}^{(N)}(t)-Y_{i}(t)\right| \\
& \leqq\left|Y_{C}^{(N)}\left(\gamma_{i}^{(N)}(t), t\right)-y_{C}\left(\gamma_{i}^{(N)}(t), t\right)\right|+\left|y_{C}\left(\gamma_{i}^{(N)}(t), t\right)-y_{C}\left(\gamma_{i}(t), t\right)\right| \\
& \leqq \sup _{\gamma \in \Gamma_{t}}\left|Y_{C}^{(N)}(\gamma, t)-y_{C}(\gamma, t)\right|+\left|y_{C}\left(\gamma_{i}^{(N)}(t), t\right)-y_{C}\left(\gamma_{i}(t), t\right)\right| .
\end{aligned}
$$

Comparing (109) and (130) we further have

$$
\begin{aligned}
& \left|Y_{i}^{(N)}(t)-Y_{i}(t)\right| \\
& \leqq \\
& \sup _{\gamma \in \Gamma_{t}}\left|Y_{C}^{(N)}(\gamma, t)-y_{C}(\gamma, t)\right| \\
& \quad+\left|y_{C}\left(\left(y_{i}^{(N)}, 0\right), t\right)-y_{C}\left(\left(y_{i}, 0\right), t\right)\right| \mathbf{1}_{t<\tau_{i, 1}}+\mathbf{1}_{\tilde{\mathcal{K}}_{i}^{(N)}(0, t)^{c}} .
\end{aligned}
$$

Substituting (136) in (137),

$$
\begin{aligned}
& \left|Y_{i}^{(N)}(t)-Y_{i}(t)\right| \\
& \leqq \sup _{\gamma \in \Gamma_{t}}\left|Y_{C}^{(N)}(\gamma, t)-y_{C}(\gamma, t)\right|+\left|y_{C}\left(\left(y_{i}^{(N)}, 0\right), t\right)-y_{C}\left(\left(y_{i}, 0\right), t\right)\right|, \quad N \geqq N_{0} .
\end{aligned}
$$

Since $y_{C} \in \Theta_{T}, \lim _{N \rightarrow \infty} y_{i}^{(N)}=y_{i}$ implies

$$
\lim _{N \rightarrow \infty} \sup _{t \in[0, T]}\left|y_{C}\left(\left(y_{i}^{(N)}, 0\right), t\right)-y_{C}\left(\left(y_{i}, 0\right), t\right)\right|=0, \text { a.s.. }
$$

Combining (134), (138), (139) we have

$$
\lim _{N \rightarrow \infty} \sup _{t \in[0, T]}\left|Y_{i}^{(N)}(t)-Y_{i}(t)\right|=0, \text { a.s.. }
$$

Therefore the almost sure uniform convergence of tagged particle system holds, which completes a proof of Theorem 2 ,

\section{A Point process with last-arrival-time dependent intensity.}

We will summarize the definition and basic formulas of the point processes with last-arrival-time dependent intensities. See [10, §3] and [11, §1.2] for a proof.

In accordance with [10, 11], we will denote the point process with last-arrival-time dependent intensity by $N=N(t)$, while we wrote $\tilde{\nu}$ in the main body of the present paper to keep the symbol $N$ for the particle number. 
Let $N=N(t), t \geqq 0$, be a non-decreasing, right-continuous, non-negative integer valued stochastic process on a measurable space with $N(0)=0$, and for each non-negative integer $k$ define its $k$-th arrival time $\tau_{k}$ by

$$
\tau_{k}=\inf \{t \geqq 0 \mid N(t) \geqq k\}, \quad k=1,2, \ldots, \quad \text { and } \tau_{0}=0 .
$$

The arrival times $\tau_{k}$ are non-decreasing in $k$, because $N$ is non-decreasing, and since $N$ is also right-continuous, the arrival times are stopping times; if we denote the associated filtration by $\mathcal{F}_{t}=\sigma[N(s), s \leqq t]$, then $\left\{\tau_{k} \leqq t\right\} \in \mathcal{F}_{t}, t \geqq 0$.

Let $\omega$ be a non-negative valued bounded continuous function of $(s, t)$ for $0 \leqq s \leqq t$, and for $k=1,2, \ldots$ assume that

$$
\mathrm{P}\left[t<\tau_{k} \mid \mathcal{F}_{\tau_{k-1}}\right]=\exp \left(-\int_{\tau_{k-1}}^{t} \omega\left(\tau_{k-1}, u\right) d u\right) \text { on } t \geqq \tau_{k-1} .
$$

In particular, (141) with $k=1$ implies

$$
\mathrm{P}[N(t)=0]=\mathrm{P}\left[\tau_{1}>t\right]=\exp \left(-\int_{0}^{t} \omega(0, u) d u\right), \quad t \geqq 0 .
$$

If $\omega$ is independent of the first variable, then (141) implies that $N$ is the (inhomogeneous) Poisson process with intensity function $\omega$. We are considering a generalization of the Poisson process such that the intensity function depends on the latest arrival time.

A construction of the point process with last-arrival-time dependent intensity goes as follows. Let $\omega:[0, \infty)^{2} \rightarrow[0, \infty)$ be a non-negative valued bounded continuous function of $(s, t)$ for $0 \leqq s \leqq t$, for which we aim to construct a process satisfying (141).

Let $\nu$ be a Poisson random measure on $[0, \infty)^{2}$, with unit constant intensity

$$
\mathrm{E}[\nu([a, b] \times[c, d])]=(b-a)(d-c) \quad b>a>0, d>c>0, \quad k \in \mathbb{N} .
$$

Define a sequence of hitting times $\tau_{k}, k \in \mathbb{Z}_{+}$, inductively by

$$
\begin{aligned}
& \tau_{0}=0, \text { and } \\
& \begin{aligned}
\tau_{k} & =\inf \left\{t \geqq \tau_{k-1} \mid \nu\left(\left\{(\xi, u) \in[0, \infty)^{2} \mid\right.\right.\right. \\
& \left.\left.\left.\quad 0 \leqq \xi \leqq \omega\left(\tau_{k-1}, u\right), \tau_{k-1}<u \leqq t\right\}\right)>0\right\} \\
k & =1,2, \ldots
\end{aligned}
\end{aligned}
$$

Note that the definition is not equivalent to the wrong formula such as $\tau_{k}=\inf \{t \geqq 0 \mid$ $\left.\nu\left(\left\{(\xi, u) \in[0, \infty)^{2} \mid 0 \leqq \xi \leqq \omega\left(\tau_{k-1}, u\right), 0<u \leqq t\right\}\right) \geqq k\right\}$. We are recursively adding 1 new arrival after the last arrival using the renewed intensity $\omega\left(\tau_{k-1}, \cdot\right)$ in (144).

$\tau_{k}$ in (141) is defined by (144), and the process $N(t)$ is defined by the reciprocal relation to (140):

$$
N(t)=\max \left\{k \in \mathbb{Z}_{+} \mid \tau_{k} \leqq t\right\}, t \geqq 0 .
$$

Since $N(t)$ and $\tau_{k}$ are samplewise non-decreasing in $t$ and $k$, respectively, (145) and (140) are equivalent. Also, (141) follows from (144).

$\left\{\tau_{k} \leqq t\right\}$ is in

$$
\mathcal{F}_{t}:=\sigma\left[\nu(A) ; A \in \mathcal{B}\left([0, \infty)^{2}\right), A \subset[0, \infty) \times[0, t], k \in \mathbb{N}\right],
$$

and consequently $N$ is adapted to $\left\{\mathcal{F}_{t}\right\}$. 
In coupling the stochastic ranking process with the flow driven stochastic ranking process, we will need a representation of $N$ by the stochastic integration with respect to $\nu$ in (143) which is,

$$
N(t)=\int_{s \in(0, t]} \int_{\xi \in[0, \infty)} \mathbf{1}_{\xi \in\left[0, \omega\left(\tau^{*}(s-), s-\right)\right)} \nu(d \xi d s), t \geqq 0,
$$

where $\tau^{*}: \Omega \times[0, \infty) \rightarrow[0, \infty)$ is the last arrival time up to time $t$ :

$$
\tau^{*}(t)=\tau_{N(t)}=\inf \{s \geqq 0 \mid N(t)=N(s)\} \in[0, t],
$$

which satisfies a stochastic integration equation

$$
\tau^{*}(t)=\int_{s \in(0, t]} \int_{\xi \in[0, \infty)}\left(s-\tau^{*}(s-)\right) \mathbf{1}_{\xi \in\left[0, \omega\left(\tau^{*}(s-), s-\right)\right)} \nu(d \xi d s),
$$

from which (147) follows.

For $t \geqq t_{0}$ put

$$
\Omega\left(t_{0}, t\right)=\int_{t_{0}}^{t} \omega\left(t_{0}, u\right) d u
$$

We have explicit formula

$$
\begin{aligned}
\mathrm{P}[ & N(t)=N(s)]=\sum_{k \geqq 0} \mathrm{P}\left[\tau_{k} \leqq s, t<\tau_{k+1}\right] \\
=\sum_{k \geqq 0} \int_{0=: u_{k}<u_{k-1}<u_{k-2}<\cdots<u_{1}<u_{0} \leqq s} & \\
& \times e^{-\sum_{i=0}^{k-1} \Omega\left(u_{i+1}, u_{i}\right)-\Omega\left(u_{0}, t\right)}\left(\prod_{i=0}^{k-1} \omega\left(u_{i+1}, u_{i}\right) d u_{i}\right),
\end{aligned}
$$

and for $\|\omega\|=\sup _{0 \leqq s \leqq t \leqq T}|\omega(s, t)|$,

$$
\begin{aligned}
& 0 \leqq-\frac{\partial}{\partial t} \mathrm{P}[N(t)=N(s)] \leqq\|\omega\|(\mathrm{P}[N(t)=N(s)]-\mathrm{P}[N(t)=N(0)]) \leqq\|\omega\|, \\
& 0 \leqq \frac{\partial}{\partial s} \mathrm{P}[N(t)=N(s)] \\
& \quad=\sum_{k \geqq 0} \int_{0=: u_{k}<u_{k-1}<\cdots<u_{1}<u_{0} \leqq s} w\left(u_{0}, s\right) \\
& \quad \times e^{-\sum_{i=0}^{k-1} \Omega\left(u_{i+1}, u_{i}\right)-\Omega\left(u_{0}, t\right)}\left(\prod_{i=0}^{k-1} \omega\left(u_{i+1}, u_{i}\right) d u_{i}\right) \\
& \\
& \leqq\|\omega\| \mathrm{P}[N(t)=N(s)] \leqq\|\omega\|, \\
& 0 \leqq
\end{aligned}
$$

\section{Acknowledgements.}

The author would like to thank Professor Seiichiro Kusuoka, Professor Kazumasa Kuwada, and Professor Naoto Miyoshi, for their interest in the present work and for giving the author opportunities for seminar talks at Okayama University, University of Tokyo, and Tokyo Institute of Technology, respectively. The author in particular thanks Prof. Kusuoka for discussions. This work is supported by JSPS KAKENHI Grant Number 26400146 from Japan Society for the Promotion of Science, and by Keio Gijuku Academic Development Funds from Keio University. 


\section{References}

[1] P. Billingsley, Convergence of probability measures, 2nd ed., John Wiley and Sons, NewYork, 1999.

[2] P. Erdös, On a Theorem of Hsu and Robbins, Ann. Math. Stat. 20 (1949) 286-291.

[3] P. Erdös, Remark on my Paper 'On a Theorem of Hsu and Robbins', Ann. Math. Stat. 21 (1950) 138.

[4] Y. Hariya, K. Hattori, T. Hattori, Y. Nagahata, Y. Takeshima, T. Kobayashi, Stochastic ranking process with time dependent intensities, Tohoku Mathematical Journal 63-1 (2011) $77-111$.

[5] K. Hattori and T. Hattori, Existence of an infinite particle limit of stochastic ranking process, Stochastic Process. Appl. 119 (2009) 966-979.

[6] K. Hattori and T. Hattori, Equation of motion for incompressible mixed fluid driven by evaporation and its application to online rankings, Funkcial. Ekvac. 52 (2009) 301-319.

[7] K. Hattori and T. Hattori, Sales ranks, Burgers-like equations, and least-recently-used caching, RIMS Kokyuroku Bessatsu B21 (2010) 149-162.

[8] T. Hattori, Solving the mystery of Amazon sales ranks (in Japanese), Kagaku Dojin, Kyoto, 2011.

[9] T. Hattori, Stochastic ranking process and web ranking numbers, in Mathematical Quantum Field Theory and Renormalization Theory, T. Hara, T. Matsui, F. Hiroshima, eds., Mathfor-Industry Lecture Note Series 30 (2011) 178-191.

[10] T. Hattori, Point process with last-arrival-time dependent intensity and 1-dimensional incompressible fluid system with evaporation, http://arxiv.org/abs/1409.5117, Funkcialaj Ekvacioj (2017), to appear.

[11] T. Hattori, Open problems to an infinite system of quasi-linear partial differential equations with non-local terms, Symposium on Probability Theory 2014/12/16-19, RIMS Kokyuroku 1952 (2015) 9-16.

[12] T. Hattori, Doubly uniform complete law of large numbers for independent point processes, preprint (2017) http://arxiv.org/abs/1612.09126.

[13] T. Hattori, S. Kusuoka, Stochastic ranking process with space-time dependent intensities, ALEA, Lat. Am. J. Probab. Math. Stat. 9(2) (2012) 571-607.

[14] P. L. Hsu, H. Robbins, Complete convergence and the law of large numbers, Proc. Nat. Acad. Sci. U.S.A. 33 (1947) 25-31.

[15] Y. Nagahata, Tagged particle dynamics in stochastic ranking process, Kodai Math. J. 36 (2013) 397-408.

[16] Y. Nagahata, Functional central limit theorem for tagged particle dynamics in stochastic ranking process, Kodai Math. J. 36 (2013) 409-427.

[17] M. L. Tsetlin, Finite automata and models of simple forms of behaviour, Russian Math. Surv. 18 (1963) 1-27. 